\title{
A new implementation of the spectral crystal plasticity framework in implicit finite elements
}

\author{
Miroslav Zecevic ${ }^{\mathrm{a}}$, Rodney J. McCabe ${ }^{\mathrm{b}}$ and Marko Knezevic ${ }^{\mathrm{a}, *}$ \\ ${ }^{a}$ Department of Mechanical Engineering, University of New Hampshire, Durham, NH 03824, USA \\ ${ }^{\mathrm{b}}$ Materials Science and Technology Division, Los Alamos National Laboratory, Los Alamos, NM 87545, USA
}

\begin{abstract}
We present a new implementation of a computationally efficient crystal plasticity model in an implicit finite element (FE) framework. In recent publications, we have reported a standalone version of a crystal plasticity model based on fast Fourier transforms (FFTs) and termed it the spectral crystal plasticity (SCP) model. In this approach, iterative solvers for obtaining the mechanical response of a single crystal of any crystallographic orientation subjected to any deformation mode are replaced by a database of FFTs that allows fast retrieval of the solution. The standalone version of the code facilitates simulations of relatively simple monotonic deformation processes under homogeneous boundary conditions. In this paper, we present a new model that enables simulations of complex, non-monotonic deformation process with heterogeneous boundary conditions. For this purpose, we derive a fully analytical Jacobian enabling an efficient coupling of SCP with implicit finite elements. In our implementation, an FE integration point can represent a single crystal or a polycrystalline material point whose mesoscale mechanical response is obtained by the mean-field Taylor-type homogenization scheme. The finite element spectral crystal plasticity (FE-SCP) implementation has been validated for several monotonic loading conditions and successfully applied to rolling and equi-channel angular extrusion deformation processes. Predictions of the FE-SCP simulations compare favorably with experimental measurements. Details of the FE-SCP implementation and predicted results are presented and discussed in this paper.
\end{abstract}

Keywords: Spectral methods; Crystal plasticity; Finite element method; UMAT; Texture; Anisotropy

"Corresponding author at: Department of Mechanical Engineering, University of New Hampshire, 33 Academic Way, Kingsbury Hall, W119, Durham, New Hampshire 03824, USA. Tel.: +1 603862 5179; fax: +1 603862 1865; E-mail address: marko.knezevic@unh.edu 


\subsection{Introduction}

As a consequence of the complex thermo-mechanical loading history experienced during their manufacture, polycrystalline metals possess a non-random distribution of grain orientations, referred to as crystallographic texture (Bunge, 1993). Texture is known to have a strong influence on the anisotropy of various material properties (Adams and Olson, 1998; FuentesCobas et al., 2013; Kocks et al., 1998). Modeling the anisotropy of plastic properties requires consideration of the crystal structure and orientation because of their role in the activation of micro-scale deformation mechanisms (Taylor, 1938). Physically-based, crystal plasticity models provide reliable predictions and help in understanding material anisotropy since they take into account microstructure and the directionality of deformation mechanisms acting at a singlecrystal level. Unlike phenomenological models based on continuum plasticity, these models can be used to optimize the microstructure and mechanical properties during production processes because they are capable of capturing evolution of the underlying microstructure during finite plastic deformation (Knezevic et al., 2012a; Knezevic et al., 2008b; Shaffer et al., 2010). As such, they are highly desirable for performing accurate simulations of metal forming processes.

A number of crystal plasticity material models linking the grain scale response to the response of a polycrystalline aggregate have been developed including the self-consistent meanfield models (Knezevic et al., 2013a; Knezevic et al., 2014a; Knezevic et al., 2013b; Lebensohn and Tomé, 1993; Lebensohn et al., 2007), Taylor type upper bound models (Knezevic et al., 2008a; Taylor, 1938; Van Houtte et al., 2004), finite-element full-field models (FE) (Kalidindi et al., 1992; Knezevic et al., 2010; Mathur et al., 1989; Roters et al., 2010; Sarma and Dawson, 1996), and Green's function fast Fourier transform (FFT) type full-field models (Lebensohn et al., 2012). While each of these homogenization approaches can be versatile in crystal mechanics and hardening laws, only the FE approach is suitable for non-monotonic deformation and treating heterogeneous boundary conditions and spatial strain gradients. Therefore, an FE implementation of crystal plasticity models is highly desirable in order to simulate complex deformation paths while retaining the micromechanical coupling of texture, stress, and strain. Example applications include simple compression and tension tests (Beaudoin et al., 1993), bending (Knezevic et al., 2013d; Knezevic et al., 2013e), rolling (Segurado et al., 2012), cupdrawing (Balasubramanian, 1996; Raabe and Roters, 2004), sheet hydroforming (Beaudoin et al., 1994), and bulk forming (Knezevic et al., 2014c; Knezevic et al., 2013c; Knezevic et al., 
2012b; Kumar and Dawson, 1995). However, the integrated crystal plasticity models within implicit FE simulations tools are computationally intensive and have not been adopted broadly by the metal forming and advanced materials development community.

Several strategies have been explored for speeding up crystal plasticity calculations. An approach relying on the adaptive sampling algorithm has been reported in (Barton et al., 2011; Barton et al., 2008). In this approach, each calculated response of a polycrystalline material point is recorded in a database on the fly as a function of input parameters. These solutions become subdomains from which subsequent model solutions can be interpolated for material points close to the already computed points instead of requiring a new call to the crystal plasticity model. Therefore, the number of direct interrogations of the intensive crystal plasticity model can be dramatically reduced. Using this approach, wall-clock speedup factors exceeding an order of magnitude relative to direct crystal plasticity calculations were demonstrated. Other attempts to improve efficiency of the crystal plasticity codes rely on a process plane concept based on proper orthogonal decomposition in Rodrigues-Frank space (Sundararaghavan and Zabaras, 2007). It has also been shown that solving crystal plasticity using the Jacobian-Free Newton-Krylov (JFNK) technique in place of the Newton-Raphson method can yield some computational benefits (Chockalingam et al., 2013).

In recent work, we have developed a computationally efficient crystal plasticity model in the spectral representation termed spectral crystal plasticity (SCP) (Knezevic et al., 2009). In the SCP framework, the crystal plasticity solutions are organized into a set of functions that describe the dependence of the stresses, the lattice spin, and the total slip rates in single crystals on their lattice orientation and the strain rate imposed on them. The domain of these functions is the product space of all possible crystal orientations and all possible isochoric strain rates. These functions were evaluated using a conventional crystal plasticity code (Kalidindi et al., 1992) and represented using Fourier bases to obtain databases of spectral coefficients. The viability of this framework was initially demonstrated using generalized spherical harmonics (GSH) bases (Fast et al., 2008; Kalidindi et al., 2006; Knezevic and Kalidindi, 2007). Subsequently, in place of the GSH bases we have explored the use of Discrete Fourier Transforms (DFTs) to generate the spectral databases (Kalidindi et al., 2009; Knezevic et al., 2009; Knezevic et al., 2008a). The major advantage of using DFTs is that the DFTs can be computed using highly efficient fast Fourier transforms (FFTs) algorithms (Briggs and Henson, 1995; Brigham, 1988; Cooley and 


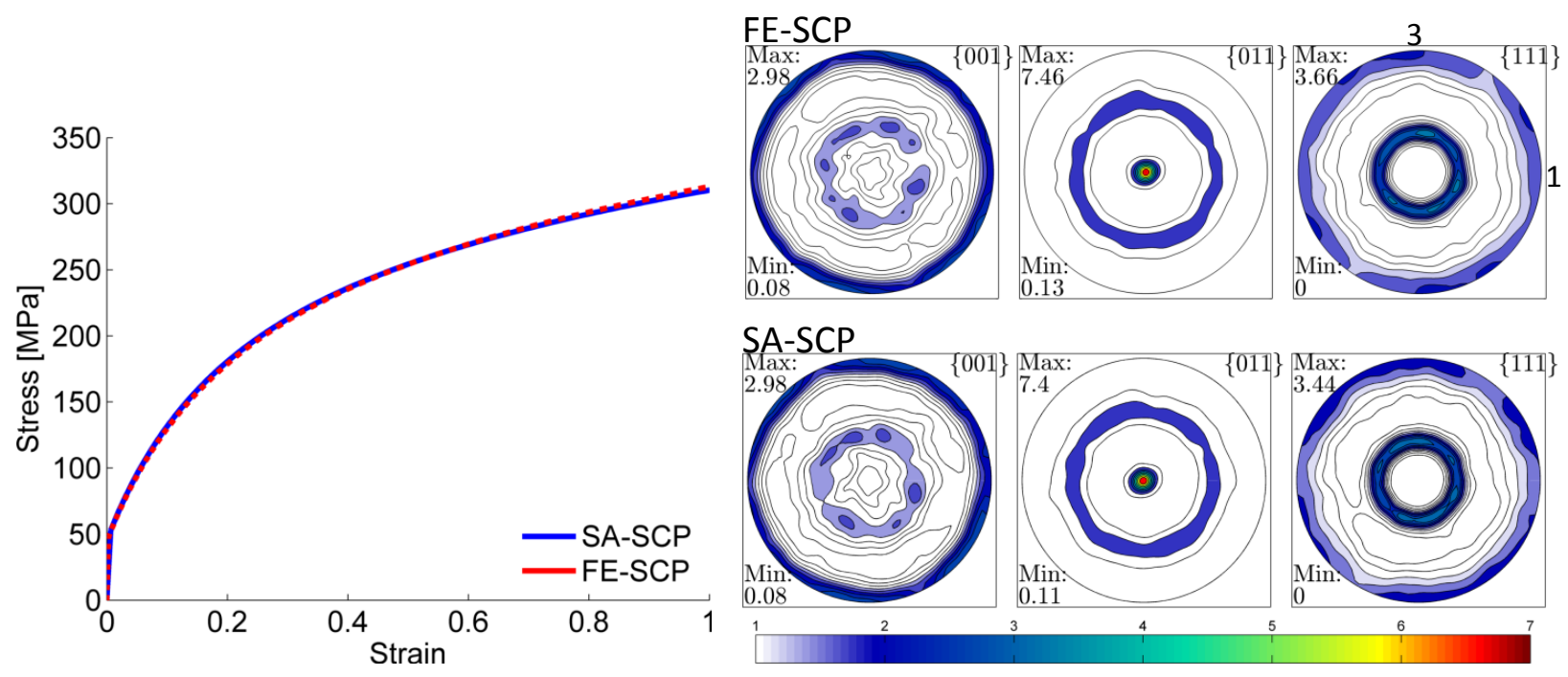

Fig. 1. Comparison of the predictions using the FE-SCP UMAT described here and the corresponding predictions using the SA-SCP model for uniaxial compression: stress-strain curves and pole figures at a strain of 1.0.

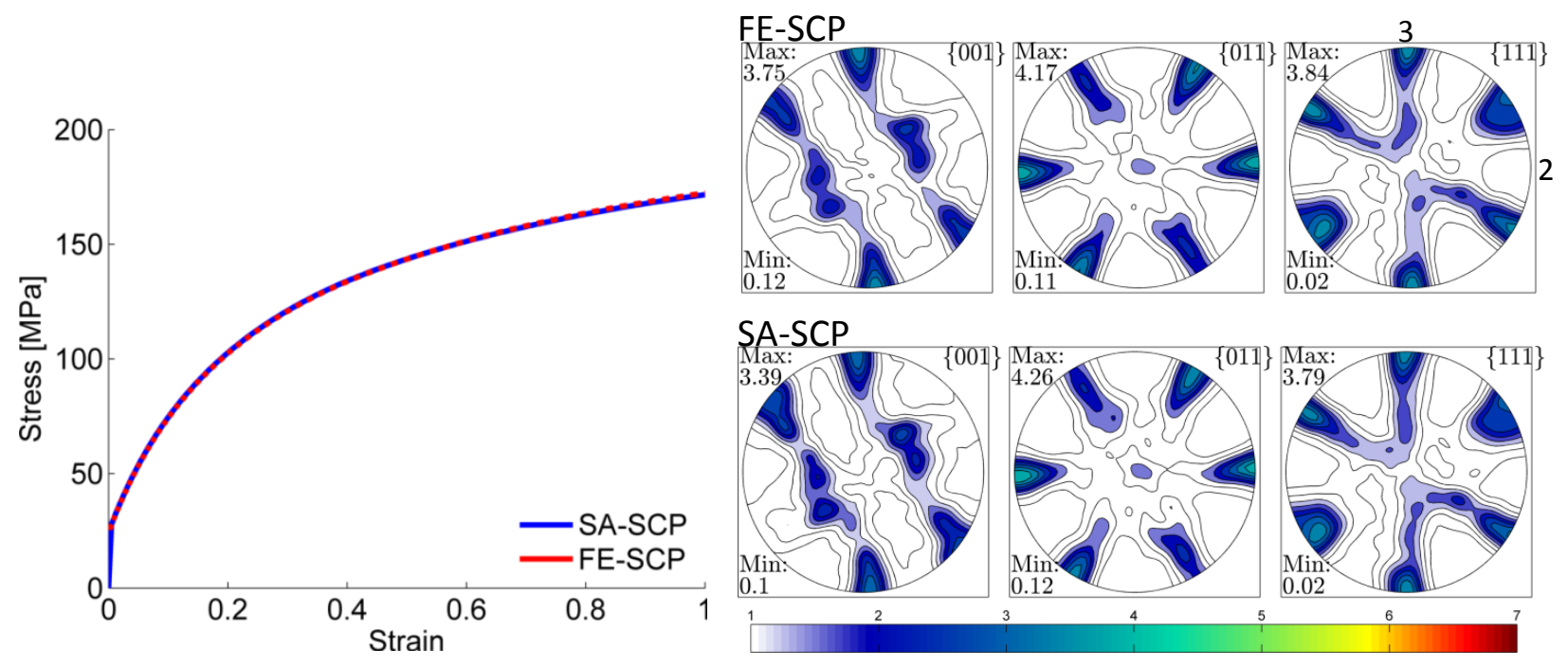

Fig. 2. Comparison of the predictions using the FE-SCP UMAT described here and the corresponding predictions using the SA-SCP model for simple shear: stress-strain curves and pole figures at a strain of 1.0 . 
Tukey, 1965; Duhamel and Vetterli, 1990; Press, 2002). The most remarkable discovery in this framework is only as few as several hundred dominant Fourier transforms are sufficient to recover the functions of interest on their respective domains. We reported speed up factors of about two orders of magnitude relative to conventional iterative solvers.

Building on these prior advances in efficient numerical schemes, we successfully developed a high performance computational application of SCP that runs on specialized hardware incorporating graphic processing units (GPUs) (Mihaila et al., 2014), and an improved version that takes advantage of an efficient GPU8 algorithm for matrix-matrix multiplication (Knezevic and Savage, 2014). The latter implementation resulted in remarkable improvements in computational speed, exceeding three orders of magnitude over the conventional numerical schemes. We have also presented a work distribution scheme on a hybrid computer architecture consisting of a cluster of Linux nodes that individually integrates multi-core processors (CPUs) and Graphics Processing Units (GPUs) intended for performing future tractable multi-scale process simulations (Knezevic and Savage, 2014). This combined framework, consisting of the computationally efficient SCP and specialized hybrid CPU-GPU hardware, has only been presented in the form of a standalone code.

The current paper is focused on a new implementation of the computationally efficient SCP framework (Al-Harbi et al., 2010; Knezevic et al., 2009; Knezevic et al., 2008a) within the implicit solver of the FE package ABAQUS through a user material subroutine (UMAT). We produce a new finite element spectral crystal plasticity (FE-SCP) model. In an implicit nonlinear FE formulation, the material constitutive model provides the stress and the tangent stiffness matrix (Jacobian). We derive and present a fully analytical Jacobian matrix ensuring fast convergence towards global stress equilibrium. The new implementation follows the main aspects of the constructive coupling presented in (Knezevic et al., 2013d; Segurado et al., 2012) and therefore differs from the first implementation presented in (Al-Harbi and Kalidindi). Moreover, the new implementation includes the hardening term in the Jacobian matrix, which we found improves the convergence. Simple compression and simple shear tests on polycrystalline copper are used to demonstrate the accuracy of the FE-SCP model in ABAQUS by comparing it with the results of the standalone SCP code. To further illustrate the capabilities and numerical stability, the FE-SCP model is applied to simulate the mechanical response and texture evolution of copper to very large strains in rolling and equi-channel angular extrusion (ECAE) tests. We 
show that the FE-VPSC simulations capture well the texture evolution during these deformations.

In Section 2, we summarize the SCP framework. In Section 3, we describe the FE-SCP implementation. In Section 4, we present validation case studies. In section 5, we present two application case studies. Finally, the conclusions are presented in Section 6.

\subsection{Summary of the spectral crystal plasticity framework}

The spectral representation for efficiently obtaining the single crystal response is summarized here using a notation that is standard in modern continuum mechanics textbooks. Tensors are denoted using bold symbols and not italic while scalars are italic and not bold. If elastic deformation is neglected, the single crystals deform according to a rate-dependent crystal plasticity theoretical model as follows. For a given crystal orientation, $g$, applied visco-plastic strain rate tensor, $\dot{\boldsymbol{\varepsilon}}_{v p}$, and prescribed unit vectors identifying the slip system direction and the slip system plane normal, $\mathbf{m}^{\alpha}$ and $\mathbf{n}^{\alpha}$ respectively, the Cauchy stress tensor deviator, $\sigma_{i j}^{\prime}\left(g, \dot{\boldsymbol{\varepsilon}}_{v p}\right)$, for slip systems $\alpha$ is calculated using the following set of equations (Asaro and Needleman, 1985; Hutchinson, 1976):

$$
\dot{\boldsymbol{\varepsilon}}_{v p}=\sum_{\alpha} \dot{\gamma}^{\alpha} \mathbf{P}^{\alpha}, \dot{\gamma}^{\alpha}=\dot{\gamma}_{o}\left|\frac{\boldsymbol{\sigma}^{\prime} \cdot \mathbf{P}^{\alpha}}{s^{\alpha}}\right|^{1 / m_{o}} \operatorname{sgn}\left(\boldsymbol{\sigma}^{\prime} \cdot \mathbf{P}^{\alpha}\right), \mathbf{P}^{\alpha}=0.5\left(\mathbf{m}^{\alpha} \otimes \mathbf{n}^{\alpha}+\mathbf{n}^{\alpha} \otimes \mathbf{m}^{\alpha}\right) .
$$

In Eq. 1, $\dot{\gamma}^{\alpha}$ and $s^{\alpha}$ represent the shearing rate and the slip resistance, respectively, on slip systems $\alpha$. The reference value of the shearing rate, $\dot{\gamma}_{o}$, is set to be $0.001 \mathrm{sec}^{-1}$ for quasi-static loading conditions. The strain rate sensitivity parameter, $m_{o}$, is 0.01 making the set of the constitutive equations in Eq. 1 extremely stiff with poor convergence characteristics. The conventional numerical procedures for solving the set of Eq. 1 for stress rely on modified Newton-Raphson schemes (e.g. Kalidindi et al., 1992) and, therefore, require considerable computational effort. The adopted modification of the Newton-Raphson scheme follows the method of Armijo for the selective reduction of the correction term (Armijo, 1966). After the shear rates on individual slip systems, $\alpha$, are known, the plastic spin contributing to the grain reorientation can easily be evaluated using:

$$
\mathbf{W}^{p}=\sum_{\alpha} 0.5 \dot{\gamma}^{\alpha}\left(\mathbf{m}^{\alpha} \otimes \mathbf{n}^{\alpha}-\mathbf{n}^{\alpha} \otimes \mathbf{m}^{\alpha}\right) .
$$


To complete this theory, a hardening law that evolves the slip resistance, $s^{\alpha}$, with accumulated plastic strain must be defined. While there are a number of hardening laws reported in the literature with various complexity from relatively simple Voce-type (Tomé et al., 1984; Voce, 1948) to complex laws based on an explicit evolution of dislocation densities (Ardeljan et al., 2014; Beyerlein et al., 2011; Knezevic et al., 2014b; Knezevic et al., 2014d), the evolution of $s^{\alpha}$, in this work, is described by an extended Voce phenomenological law (Voce, 1948) expressed as

$$
\dot{s}^{\alpha}=h_{o}\left(1-\frac{s^{\alpha}}{s_{s}}\right)^{a} \sum_{\beta}\left|\dot{\gamma}^{\beta}\right|,
$$

where $h_{o}, s_{s}$ and $a$ denote the slip hardening parameters. This law assumes that the slip resistance is the same for all slip systems within a grain but varies from grain-to-grain. The values of the slip hardening parameters were calibrated for pure copper using the Taylor-type model in (Kalidindi et al., 1992) and used in this work. The values are $h_{o}=180 \mathrm{MPa}, s_{s}=148 \mathrm{MPa}, a=2.25$, and $s_{o}=16 \mathrm{MPa}$, where $s_{o}$ denotes initial value of the slip resistance.

As remarked earlier, the conventional iterative procedures demand an extremely high computational effort and are impractical for large scale simulations because the sets of equations defined in Eq. 1 have to be solved for every constituent crystal at every material point and at each trial time increment. In addition, since every material point under consideration has many individual crystals, it is expected that the same or similar computations are repeated over and over in a crystal plasticity simulation because the results of computations in any one time step are immediately disregarded once the computations advance to the next time step. The question at hand is whether it is possible to capture the solutions from the crystal plasticity models in a database, such that the necessary computations are performed once and the results are stored and can be reused in any subsequent calculations. This approach circumvents the iterative Newton's methods. The added benefit is a compact database and an efficient retrieval methodology of solutions. We now turn our attention to the main aspects of spectral crystal plasticity.

In the spectral crystal plasticity approach (Knezevic et al., 2009), we establish efficient spectral representations for the essential functions capturing the solutions to the crystal plasticity theory described above. The representation is based on fast Fourier transforms (FFTs). The 
functions are components of the Cauchy stress deviator, $\sigma_{i j}^{\prime}\left(g, \dot{\boldsymbol{\varepsilon}}_{v p}\right)$, the plastic lattice spin, $W_{i j}^{p}\left(g, \dot{\boldsymbol{\varepsilon}}_{v p}\right)$, and summation of the slip rates, $\sum_{\alpha}\left|\dot{\gamma}^{\alpha}\right|\left(g, \dot{\boldsymbol{\varepsilon}}_{v p}\right)$, as functions of the crystal lattice orientation, $g$, and the applied strain rate tensor, $\dot{\boldsymbol{\varepsilon}}_{v p}$. The domain of the functions is, therefore, the Bunge-Euler space and the strain rate space. We now show that this space is four dimensional (4D).

The applied visco-plastic strain rate tensor, $\dot{\boldsymbol{\varepsilon}}_{v p}$, is assumed to be isochoric meaning that the zero-trace condition is applied to $\dot{\boldsymbol{\varepsilon}}_{v p}$. Therefore, $\dot{\boldsymbol{\varepsilon}}_{v p}$ has five independent components. In metal plasticity, the plastic deformation is usually assumed to be isochoric, i.e. fully incompressible, and the compressible part is a result of elastic deformations only, which remain relatively small during large plastic deformation. It is now possible to parameterize $\dot{\boldsymbol{\varepsilon}}_{v p}$ using a single angular variable, $\theta$, in its principal frame, $\left\{\tilde{\mathbf{e}}_{i}, i=1,2,3\right\}$, (Van Houtte, 1994) as follows:

$$
\begin{aligned}
& \tilde{\dot{\boldsymbol{\varepsilon}}}_{v p}=\dot{\varepsilon}_{v p} \hat{\dot{\boldsymbol{\varepsilon}}}_{v p}, \quad \hat{\boldsymbol{\varepsilon}}_{v p}=\sum_{j=1}^{3} \dot{\lambda}_{j} \tilde{\mathbf{e}}_{j} \otimes \tilde{\mathbf{e}}_{j}, \quad \dot{\varepsilon}_{v p}=\left|\dot{\boldsymbol{\varepsilon}}_{v p}\right|, \quad \hat{\dot{\boldsymbol{\varepsilon}}}_{v p}=\frac{\tilde{\dot{\boldsymbol{\varepsilon}}}_{v p}}{\left|\tilde{\dot{\boldsymbol{\varepsilon}}}_{v p}\right|}, \\
& \dot{\lambda}_{1}=\sqrt{\frac{2}{3}} \cos \left(\theta-\frac{\pi}{3}\right), \quad \dot{\lambda}_{2}=\sqrt{\frac{2}{3}} \cos \left(\theta+\frac{\pi}{3}\right), \quad \dot{\lambda}_{3}=-\sqrt{\frac{2}{3}} \cos (\theta) .
\end{aligned}
$$

We place the tilde grapheme, " ", on top of all variables expressed in the principal frame of $\dot{\boldsymbol{\varepsilon}}_{v p}$. The principal frame is defined by the principal vectors of $\dot{\boldsymbol{\varepsilon}}_{v p}$. The principal frame and the reference sample frame are then related by the transformation matrix, $\mathbf{Q}=\left[\left\{\tilde{\mathbf{e}}_{1}\right\}\left\{\tilde{\mathbf{e}}_{2}\right\}\left\{\tilde{\mathbf{e}}_{3}\right\}\right]$, whose columns are normalized principal vectors of $\dot{\boldsymbol{\varepsilon}}_{v p}$. Thus, the applied strain rate tensor in the principal frame is $\widetilde{\boldsymbol{\varepsilon}}_{v p}=\mathbf{Q}^{T} \dot{\boldsymbol{\varepsilon}}_{v p} \mathbf{Q}$. Our objective is to reduce $\dot{\boldsymbol{\varepsilon}}_{v p}$ to a single angular variable, $\theta$, in the range $[0,2 \pi)$. In this range, the angular variable, $\theta$, can describe all possible diagonal matrices (Van Houtte, 1994). Five components of $\dot{\boldsymbol{\varepsilon}}_{v p}$ are sufficient to describe any isochoric visco-plastic deformation. The reduction from five to four components is achieved by extracting the norm of the $\dot{\boldsymbol{\varepsilon}}_{v p}$ tensor and defining a unit visco-plastic strain rate tensor in the principal frame. In Eq. 4, the norm is denoted by the scalar quantity $\dot{\varepsilon}_{v p}$ and the unit visco-plastic strain 
rate in the principal frame by the tensorial quantity $\hat{\boldsymbol{\varepsilon}}_{v p}$. The unit visco-plastic strain rate tensor is used for building the database of the spectral coefficients. Finally, $\hat{\boldsymbol{\varepsilon}}_{v p}$ can be represented by a single variable, $\theta$, just like any other diagonal matrix (Van Houtte, 1994). The remaining three variables are principal vectors that comprise a transformation matrix relating the principal to sample frame, $\mathbf{Q}=\left[\left\{\tilde{\mathbf{e}}_{1}\right\}\left\{\tilde{\mathbf{e}}_{2}\right\}\left\{\tilde{\mathbf{e}}_{3}\right\}\right]$. The transformation matrix can be described using any set of three independent variables describing an orientation, i.e. a set of Bunge-Euler angles, an angleaxis pair (Frank, 1987), a Rodriguez vector (Neumann, 1991), and a set of quaternions (Takahashi et al., 1985). Consequently, the functions we seek are expressed in terms of the following five variables $(\theta, \dot{\varepsilon}, \mathbf{Q})$, instead of the five components of $\dot{\boldsymbol{\varepsilon}}_{v p}$. The crystal plasticity calculations are performed in the $\left\{\tilde{\mathbf{e}}_{i}, i=1,2,3\right\}$ frame and transformed back to the sample reference frame, $\left\{\mathbf{e}_{i}, i=1,2,3\right\}$, using $\mathbf{Q}$ whenever an output is needed. In our notation, $g$ denotes the crystal orientation with respect to the $\left\{\mathbf{e}_{i}, i=1,2,3\right\}$ frame and $\tilde{g}$ denotes the crystal orientation with respect to the $\left\{\tilde{\mathbf{e}}_{i}, i=1,2,3\right\}$ frame. Texture evolution during simulations is kept in the principal frame but is output in the sample frame. The same holds for stress, i.e. $\boldsymbol{\sigma}^{\prime}=\mathbf{Q} \tilde{\boldsymbol{\sigma}}^{\prime} \mathbf{Q}^{T}$. The norm, $\dot{\varepsilon}_{v p}$, is explicitly contained in spectral equations for the stress, grain spin, and summation of shearing rate.

The spectral database of conventional crystal plasticity is obtained by discretizing the functions $\tilde{\boldsymbol{\sigma}}(\tilde{g}, \theta), \tilde{\mathbf{W}}^{p}(\tilde{g}, \theta)$, and $\sum_{\alpha}\left|\dot{\gamma}^{\alpha}\right|(\tilde{g}, \theta)$ on a uniform grid in the product space defined by the crystal-orientation space, $\widetilde{g}$, (Bunge, 1993) and the strain rate space, $\theta$ : four angular variables running from 0 to 360 degrees. These field variables are computed for each crystal orientation from Euler space subjected to all strain rates. The numerical values of these functions are computed using the conventional single-crystal plasticity theory described in (Kalidindi et al., 1992). Then, the spectral representation is obtained by Fourier transforming the computed functions:

$$
\begin{aligned}
& \widetilde{\boldsymbol{\sigma}}_{r s t q}^{\prime}=s \dot{\varepsilon}_{v p}^{m_{o}} \frac{1}{N_{\varphi_{1} N_{\phi} N_{\varphi_{2}} N_{\theta}}} \sum_{k} \sum_{l} \sum_{m} \sum_{n} \mathbf{F}_{k l m n} e^{\frac{2 \pi i k r}{N \varphi_{1}}} e^{\frac{2 \pi i l s}{N_{\phi}}} e^{\frac{2 \pi i m t}{N_{\varphi_{2}}}} e^{\frac{2 \pi i n q}{N_{\theta}}}, \\
& \widetilde{\mathbf{W}}_{r s t q}^{p}=\dot{\varepsilon}_{v p} \frac{1}{N_{\varphi_{1} N_{\phi} N_{\varphi_{2}} N_{\theta}}} \sum_{k} \sum_{l} \sum_{m} \sum_{n} \mathbf{B}_{k l m n} e^{\frac{2 \pi i k r}{N_{\varphi_{1}}}} e^{\frac{2 \pi i l s}{N_{\phi}}} e^{\frac{2 \pi i m t}{N_{\varphi_{2}}}} e^{\frac{2 \pi i n q}{N_{\theta}}}
\end{aligned}
$$




$$
\left(\sum_{\alpha}\left|\dot{\gamma}^{\alpha}\right|\right)_{r s t q}=\dot{\varepsilon}_{v p} \frac{1}{N_{\varphi_{1}} N_{\phi} N_{\varphi_{2}} N_{\theta}} \sum_{k} \sum_{l} \sum_{m} \sum_{n} G_{k l m n} e^{\frac{2 \pi i k r}{N_{\varphi_{1}}}} e^{\frac{2 \pi i l s}{N_{\phi}}} e^{\frac{2 \pi i m t}{N_{\varphi_{2}}}} e^{\frac{2 \pi i n q}{N_{\theta}}}
$$

where $N_{\varphi_{1}}, N_{\phi}, N_{\varphi_{2}}$ and $N_{\theta}$ are the total number of grid points in the orientation space, $\widetilde{g}$, and deformation space, $\theta$, used during sampling of function values. Equations 5-7 recover functions in the principal frame of the stretching tensor for any given $\tilde{g}$ (indexed by $r, s$, and $t$ ) and $\tilde{\dot{\boldsymbol{\varepsilon}}}_{v p}$ (indexed by $q$ ).

The sets of coefficients $\mathbf{F}_{k l m n}, \mathbf{C}_{k l m n}$ and $G_{k l m n}$ are evaluated using standard fast Fourier transform (FFT) algorithms on a three degree grid in each dimension of the four dimensional space. Therefore $120^{4}$ discrete values are obtained for each function in the real space. The number of spectral coefficients is as large as the number of function values in real space, however, the magnitude of these spectral amplitudes decreases exponentially (Knezevic et al., 2009). Therefore, we apply a thresholding procedure and disregard all spectral amplitudes with magnitudes lower than the threshold magnitude value. We set the required threshold value such that we keep 1024 Fourier transforms. Power of 2 was selected because it yields better performance involving FFT calculations. We established that 1024 transforms accurately capture the representation of the essential functions and that the predicted stress-strain response and texture evolution using SCP compare favorably with those predicted by the conventional iterative code used to build the database. In any given time step in the simulation of the deformation process, these functions can then be used to compute all of the needed microscale (i.e. grain scale stress, grain spin, and shearing rates on slip systems) and macroscale (i.e. homogenized stress-strain response and texture) quantities that are typically computed by traditional crystal plasticity approaches. Here, we evaluate a series instead of going through an iterative solution procedure.

The above summarized model can be used to predict the stress-strain response and the texture evolution of single or polycrystalline materials after applying strain increment steps. Given a set of crystal orientations, an initial value of slip resistance per crystal orientation, and an imposed value of the $\dot{\boldsymbol{\varepsilon}}_{v p}$ tensor; the stress tensor, the spin tensor and the summation of the slip rate can be evaluated for each crystal orientation using Eqs. 5-7. The spin tensor of individual orientations is used for their reorientation. The updated set of crystal orientations enters the next strain increment. Additionally, the summation of slip rate is used to update the slip resistance using Eq. 
3 for the next strain increment. For a polycrystalline response, we use the Taylor model, in which the applied strain rate tensor at the grain level is assumed to be the same as the one applied at the polycrystal level. The macroscopic stress for the polycrystal is then obtained by volume averaging the stresses inside the polycrystal. The standalone SCP, (SA-SCP) code can be used in simulations under homogeneous boundary conditions. In order to use it within the implicit FE framework and enable treating any heterogeneous boundary conditions, it is necessary to derive the Jacobian matrix. Details of the coupling of SCP with FE are presented in the next section.

\subsection{Finite element spectral crystal plasticity UMAT}

FE-SCP is implemented as a User Material Subroutine (UMAT) in ABAQUS Standard and each finite element integration point in a finite element mesh can be associated with a specific single crystal or a polycrystalline material. In the former case, the overall response of the polycrystalline material is obtained by the micromechanical finite element homogenization. In the latter case, we calculate a meso-scale mechanical response using the Taylor-type homogenization. In both cases, the overall finite element models satisfy equilibrium and compatibility conditions in the weak numerical sense. The applied load is divided into increments, and the equilibrium at each increment is obtained by means of the $\mathrm{FE}$ analysis in an iterative fashion using a global nonlinear solver with the load increment controlled by time. For each element, the linearized equilibrium FE equation based on the principal of virtual work is:

$$
\left(\int_{V} \overline{\mathbf{B}}^{\mathrm{T}} \mathbf{J} \overline{\mathbf{B}} d V\right) \Delta \mathbf{U}={ }^{\tau} \mathbf{f}-\int_{V} \overline{\mathbf{B}}^{\mathrm{T}} \boldsymbol{\sigma} d V .
$$

The left-hand side consists of the strain-displacement matrix, $\overline{\mathbf{B}}$, material Jacobian, $\mathbf{J}=\partial \Delta \boldsymbol{\sigma} / \partial \Delta \boldsymbol{\varepsilon}$, and displacement increment solution field, $\Delta \mathbf{U}$, while the right-hand side represents the residual that includes the Cauchy stress, $\boldsymbol{\sigma}$, and any applied forces, f, (Bathe, 1996). Note that from an FE code, the strain increment, $\Delta \boldsymbol{\varepsilon}^{\mathrm{FE}}$, the rotation increment, $\Delta \mathbf{R}$, at current $t+\Delta t$, the stress, $\boldsymbol{\sigma}^{t}$, and the set of internal state variables including crystal orientation corresponding to the previous time increment, $t$, and time increment, $\Delta t$, are passed to the material subroutine for each integration point. The user material model provides the Cauchy stresses, $\boldsymbol{\sigma}$, the Jacobian, $\mathbf{J}$, and updated material state variables for each integration point at each trial time increment. When convergence in stress equilibrium is achieved by the global 
nonlinear scheme, the new values (at $t+\Delta t$ ) of $\boldsymbol{\sigma}$, the internal variables, and the Jacobian matrix are accepted for every integration point, and the calculation advances to the next increment.

For a given $\Delta \varepsilon^{\mathrm{FE}}$, the FE-SCP UMAT is based on the minimization procedure used earlier in the incorporation of the visco-plastic self-consistent model in implicit finite elements (Knezevic et al., 2013d; Segurado et al., 2012) and is as follows. The total strain increment, $\Delta \boldsymbol{\varepsilon}=\Delta \boldsymbol{\varepsilon}^{\mathrm{FE}}$, is divided into the elastic, $\Delta \boldsymbol{\varepsilon}_{e l}$, and viscoplastic, $\Delta \boldsymbol{\varepsilon}_{v p}$, parts as:

$$
\Delta \boldsymbol{\varepsilon}=\Delta \boldsymbol{\varepsilon}_{e l}+\Delta \boldsymbol{\varepsilon}_{v p}=\mathbf{S} \cdot \Delta \boldsymbol{\sigma}+\dot{\boldsymbol{\varepsilon}}_{v p} \Delta \mathbf{t}
$$

where $\mathbf{S}$ is the four rank elastic compliance tensor of the material point, $\Delta \boldsymbol{\sigma}$ is the Cauchy stress increment, and $\Delta \boldsymbol{\varepsilon}_{v p}=\Delta \boldsymbol{\varepsilon}_{v p}\left(\boldsymbol{\sigma}^{\prime}\right)$ is computed using the SCP model. Note that $\boldsymbol{\sigma}=\boldsymbol{\sigma}^{\prime}+\sigma_{m} \mathbf{I}$, where $\sigma_{m}$ is the hydrostatic stress and $\mathbf{I}$ is an identity matrix. The material derivative of Cauchy stress in the global frame is given by (Asaro and Needleman, 1985; Hill, 1966; Peirce et al., 1982):

$$
\dot{\boldsymbol{\sigma}}=\boldsymbol{\sigma}^{*}+\mathbf{W}^{*} \boldsymbol{\sigma}-\boldsymbol{\sigma} \mathbf{W}^{*} \text {. }
$$

In Eq. (10), $\boldsymbol{\sigma}^{*}=\mathbf{C} \cdot\left(\dot{\boldsymbol{\varepsilon}}-\dot{\boldsymbol{\varepsilon}}_{v p}\right)$ is the lattice corotational Cauchy stress rate, which is related to the elastic strain-rate through elastic stiffness, $\mathbf{C}$. The elastic compliance, $\mathbf{S}$, used later, is calculated as the inverse of $\mathbf{C}$. The elastic properties are calculated based on the elastic single crystal constants for copper, $C_{11}=168.4 \mathrm{GPa}, C_{12}=121.4 \mathrm{GPa}$, and $C_{44}=75.4 \mathrm{GPa}$ (Meyers and Chawla, 1998) at the beginning of each deformation increment and the textural changes are accounted for in determining the elastic properties. The constitutive relation for the Cauchy stress required by $\mathrm{ABAQUS}$ at the material point at $t+\Delta t$ is given by:

$$
\boldsymbol{\sigma}^{t+\Delta t}=\boldsymbol{\sigma}^{t, r o t}+\boldsymbol{\sigma}^{*} \Delta t=\boldsymbol{\sigma}^{t, r o t}+\mathbf{C} \cdot\left(\Delta \boldsymbol{\varepsilon}-\dot{\boldsymbol{\varepsilon}}_{v p}^{t+\Delta t} \Delta t\right) .
$$

Note that the stress tensor, $\boldsymbol{\sigma}^{t}$, is rotated according to $\boldsymbol{\sigma}^{t, r o t}=\boldsymbol{\sigma}^{t}+\int_{t}^{t+\Delta t}\left(\mathbf{W}^{*} \boldsymbol{\sigma}-\boldsymbol{\sigma} \mathbf{W}^{*}\right) d \tau=\Delta \mathbf{R}^{*} \boldsymbol{\sigma}^{t} \Delta \mathbf{R}^{*}$ (Hughes and Winget, 1980). It should be noted that all variables in Eq. (11) are in the global reference frame, as required by ABAQUS (ABAQUS User Reference Manual (2013)). Using Eq. (11) and noting that $\boldsymbol{\sigma}=\boldsymbol{\sigma}^{\prime}+\sigma_{m} \mathbf{I}$, we define the residual $\mathbf{X}\left(\dot{\boldsymbol{\varepsilon}}_{v p}^{t+\Delta t}, \sigma_{m}^{t+\Delta t}\right)$ at each material point as a nonlinear function of the strain rate, $\dot{\boldsymbol{\varepsilon}}_{v p}^{t+\Delta t}$, and hydrostatic stress, $\sigma_{m}^{t+\Delta t}$, in the vector form: 


$$
\mathbf{X}\left(\dot{\boldsymbol{\varepsilon}}_{v p}^{t+\Delta t}, \boldsymbol{\sigma}_{m}^{t+\Delta t}\right)=\boldsymbol{\sigma}^{t+\Delta t}\left(\dot{\boldsymbol{\varepsilon}}_{v p}^{t+\Delta t}\right)+\sigma_{m}^{t+\Delta t} \mathbf{I}-\boldsymbol{\sigma}^{t, r o t}-\mathbf{C}\left(\Delta \boldsymbol{\varepsilon}-\dot{\boldsymbol{\varepsilon}}_{v p}^{t+\Delta t} \Delta \mathrm{t}\right)
$$

The condition $\mathbf{X}\left(\dot{\boldsymbol{\varepsilon}}_{v p}^{t+\Delta t}, \sigma_{m}^{t+\Delta t}\right)=\mathbf{0}$ is enforced using an iterative Newton's method of solving the nonlinear system of equations for $\dot{\boldsymbol{\varepsilon}}_{v p}^{t+\Delta t}, \sigma_{m}^{t+\Delta t}$. In doing so, we organize the variables in Eq. (12) in a vector form as $\mathbf{Y}=\left\{\dot{\varepsilon}_{v p, 11}^{t+\Delta t}, \dot{\varepsilon}_{v p, 22}^{t+\Delta t}, \dot{\varepsilon}_{v p, 12}^{t+\Delta t}, \dot{\varepsilon}_{v p, 13}^{t+\Delta t}, \dot{\varepsilon}_{v p, 23}^{t+\Delta t}, \sigma_{m}^{t+\Delta t}\right\}=\left\{\dot{\mathbf{e}}_{v p}^{t+\Delta t}, \sigma_{m}^{t+\Delta t}\right\}$, $\dot{\varepsilon}_{v p}^{t+\Delta t}=\left\{\dot{\varepsilon}_{v p, 11}^{t+\Delta t}, \quad \dot{\varepsilon}_{v p, 22}^{t+\Delta t},-\left(\dot{\varepsilon}_{v p, 11}^{t+\Delta t}+\dot{\varepsilon}_{v p, 22}^{t+\Delta t}\right), 2 \dot{\varepsilon}_{v p, 12}^{t+\Delta t}, 2 \dot{\varepsilon}_{v p, 13}^{t+\Delta t}, 2 \dot{\varepsilon}_{v p, 23}^{t+\Delta t}\right\}$

$\boldsymbol{\sigma}^{\prime t+\Delta t}=\left\{{\sigma^{\prime}}_{11}^{t+\Delta t},{\sigma^{\prime}}_{22}^{t+\Delta t},-\left({\sigma^{\prime}}_{11}^{t+\Delta t}+{\sigma^{\prime}}_{22}^{t+\Delta t}\right),{\sigma^{\prime}}_{12}^{t+\Delta t}, \sigma_{13}^{\prime t+\Delta t}, \sigma_{23}^{\prime t+\Delta t}\right\}, \quad$ and $\quad \mathbf{i}=\{1,1,1,0,0,0\}$. Hence, given a guess, $\mathbf{Y}^{k-1}$, the new guess is obtained as:

$$
\mathbf{Y}^{k}=\mathbf{Y}^{k-1}-\mathbf{J}_{N R}^{-1}\left(\mathbf{Y}^{k-1}\right) \mathbf{X}\left(\mathbf{Y}^{k-1}\right)
$$

where,

$$
\mathbf{J}_{N R}=\frac{\partial \mathbf{X}}{\partial \mathbf{Y}}=\left[\left[\frac{\partial \boldsymbol{\sigma}^{\prime}}{\partial \dot{\mathbf{e}}_{v p}}+\mathbf{C} \frac{\partial \dot{\boldsymbol{\varepsilon}}_{v p}}{\partial \dot{\mathbf{e}}_{v p}} \Delta t\right]_{6 \times 5}, \frac{\partial \mathbf{X}}{\partial \sigma_{m}}\right]_{6 \times 6}, \frac{\partial \mathbf{X}}{\partial \sigma_{m}}=\mathbf{i}
$$

Solving Eq. 12 typically takes from 2 to 5 iterations with a possible higher number of iterations during the elasto-plastic transition or unloading. Note that there is a linear dependence between $\mathbf{X}$ and $\sigma_{m}$ but nonlinear between $\mathbf{X}$ and $\dot{\mathbf{e}}_{v p}$. From Eq. (9), we obtain a closed form expression for the Jacobian matrix that the FE-SCP UMAT needs to return to ABAQUS as:

$$
\mathbf{J}=\frac{\partial \Delta \boldsymbol{\sigma}}{\partial \Delta \varepsilon}=\left(\frac{\partial \Delta \varepsilon}{\partial \Delta \boldsymbol{\sigma}}\right)^{-1}=\left(\mathbf{S}+\frac{\partial \dot{\boldsymbol{\varepsilon}}_{v p}}{\partial \Delta \boldsymbol{\sigma}} \Delta t\right)^{-1}=\left(\mathbf{S}+\frac{\partial \dot{\boldsymbol{\varepsilon}}_{v p}}{\partial \dot{\mathbf{e}}_{v p}}\left(\frac{\partial \boldsymbol{\sigma}^{\prime}}{\partial \dot{\mathbf{e}}_{v p}}\right)^{-1} \frac{\partial \boldsymbol{\sigma}^{\prime}}{\partial \boldsymbol{\sigma}} \Delta t\right)^{-1}
$$

Overlap between several terms in Eqs. (14) and (15) occurs, which greatly reduces the overall computational cost. Details of the fully analytical expressions for $\frac{\partial \boldsymbol{\sigma}}{\partial \boldsymbol{\sigma}^{\prime}}$ and $\frac{\partial \boldsymbol{\sigma}^{\prime}}{\partial \dot{\mathbf{e}}_{v p}}$ are presented in Appendix A and Appendix B, respectively. In Eq. (15), vector $\boldsymbol{\sigma}^{\prime}$ contains five independent components of deviatoric stress and, therefore, the size of the matrices in the chain are $6 \times 5,5 \times 5$, and 5x6. Appendix C presents the overall algorithm of the FE-SCP UMAT implementation.

The difference between the tangent stiffness matrix for a single crystal and a polycrystalline aggregate embedded at an integration point is that the later requires calculation of the average value over constituent crystals. Since the Taylor-type homogenization is used, a polycrystalline tangent stiffness is calculated based on the averaged values of $\mathbf{S}$ and $\frac{\partial \boldsymbol{\sigma}^{\prime}}{\partial \dot{\mathbf{e}}_{v p}}$ in Eq. 15.

The above procedure is fully implicit in the values of stress and slip resistances, where internal equilibrium is checked at every iteration. The internal variables (a single orientation or a set of orientations described by Bunge-Euler angles) are updated at the end of the increment. 
As a final note, we reflect on the imposed rotation rate. A single crystal or a polycrystalline material embedded at an FE integration point undergoes macroscopic rotation due to the macroscopically-imposed spin $\mathbf{W}$ at every time increment. This spin and the plastic spin, $\mathbf{W}^{p}$, define the lattice spin, $\mathbf{W}^{*}=\mathbf{W}-\mathbf{W}^{p}$, responsible for texture evolution. Therefore, each single crystal undergoes reorientation due to $\mathbf{W}$ in addition to a reorientation due to the plastic spin, $\mathbf{W}^{p}$. The macroscopically-imposed spin, $\mathbf{W}$, is calculated from the increment of the macroscopic rotation, $\Delta \mathbf{R}$, provided by ABAQUS as $\mathbf{W}=\mathbf{N} \dot{\theta}$, where $\mathbf{N}$ is the antisymmetric tensor expressed in terms of a dual vector $\mathbf{n}$ whose components are obtained from $\Delta \mathbf{R}$ as follows: $n_{1}=\Delta R_{32}-\Delta R_{23}, n_{2}=\Delta R_{13}-\Delta R_{31}, n_{3}=\Delta R_{21}-\Delta R_{12}$ and $\dot{\theta}=\arccos \left(1 / 2\left(\Delta R_{i i}-1\right)\right) / \Delta t$. The components of the dual vector must be normalized to make it a unit vector. The field and state variables are sent back to ABAQUS in the global frame.

\section{Validation of the FE-SCP UMAT}

In the following we present several case studies used to test the accuracy of the FE-SCP model in ABAQUS. First, we compare directly the stress-strain response and texture predicted by the FE-SCP model against the results obtained using the stand-alone SA-SCP model. Next, we predict geometrical changes of a single crystal with Goss orientation under compression and compare it with available data in literature. Finally, we apply the model to simulate gradients in texture evolution under rolling and equi-channel angular extrusion and also compare these predictions with available data in the literature.

\subsection{Comparison of the predictions of FE-SCP with SA-SCP}

Here, we compare predictions of the FE-SCP UMAT with the prediction of the SA-SCP code for two case studies. Specifically we compare the simulated stress-strain response and texture evolution under uniaxial compression and simple shear deformation to a strain of 1.0. The initial texture was assumed to be random and represented using 512 crystal orientations. These two case studies were selected because both SA-SCP and FE-SCP independently can handle simulations with such boundary conditions. We constructed simple FE models containing one linear element C3D8 (8 nodes, and 8 integration points each considering the same 512 crystal orientations) with displacements imposed along the $\mathbf{e}_{3}$ direction for the compression and stress- 
free boundary conditions on the lateral faces. The same test was simulated with the SA-SCP model. Comparison of the predicted mechanical response and texture evolution is shown in Fig. 1 and excellent agreement is obtained. Similarly, we performed the simple shear tests using both the SA-SCP model as well as the FE-SCP UMAT using the same one element model. We use the following deformation gradient $\mathbf{F}=\mathbf{I}+\gamma \mathbf{e}_{2} \otimes \mathbf{e}_{3}$ in both models, with $\gamma=2.0$. The simple shear analysis was performed to verify calculations of the imposed spin on texture evolution. Note that the imposed spin during uniaxial compression is zero. The predicted stress-strain curves and pole figures predicted by the two models are in excellent agreement (see Fig. 2). Note that the SASCP model does not account for any elastic deformation, which causes some negligible differences in comparison with the FE-SCP UMAT predictions.

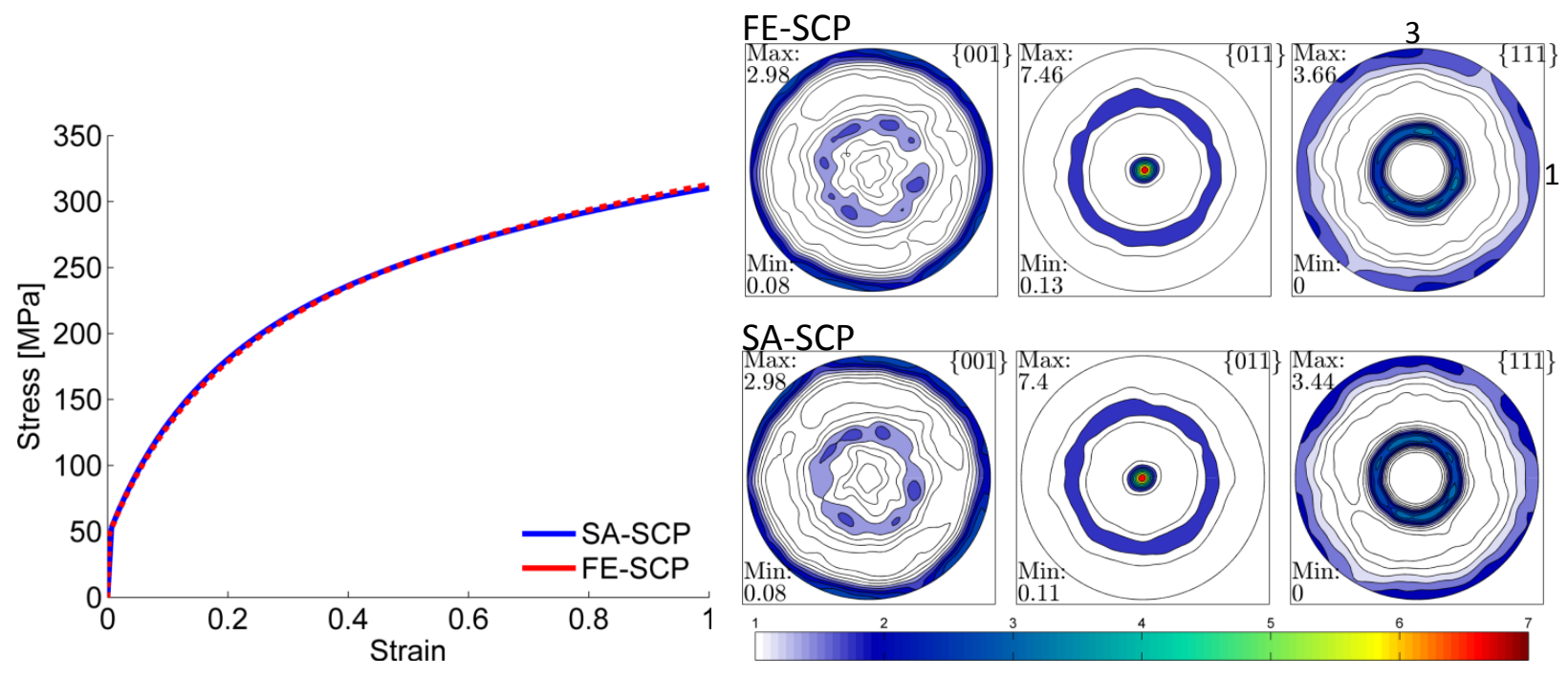

Fig. 1. Comparison of the predictions using the FE-SCP UMAT described here and the corresponding predictions using the SA-SCP model for uniaxial compression: stress-strain curves and pole figures at a strain of 1.0.
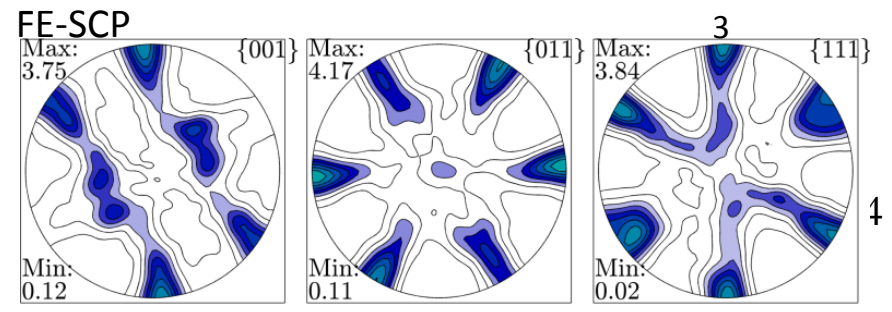


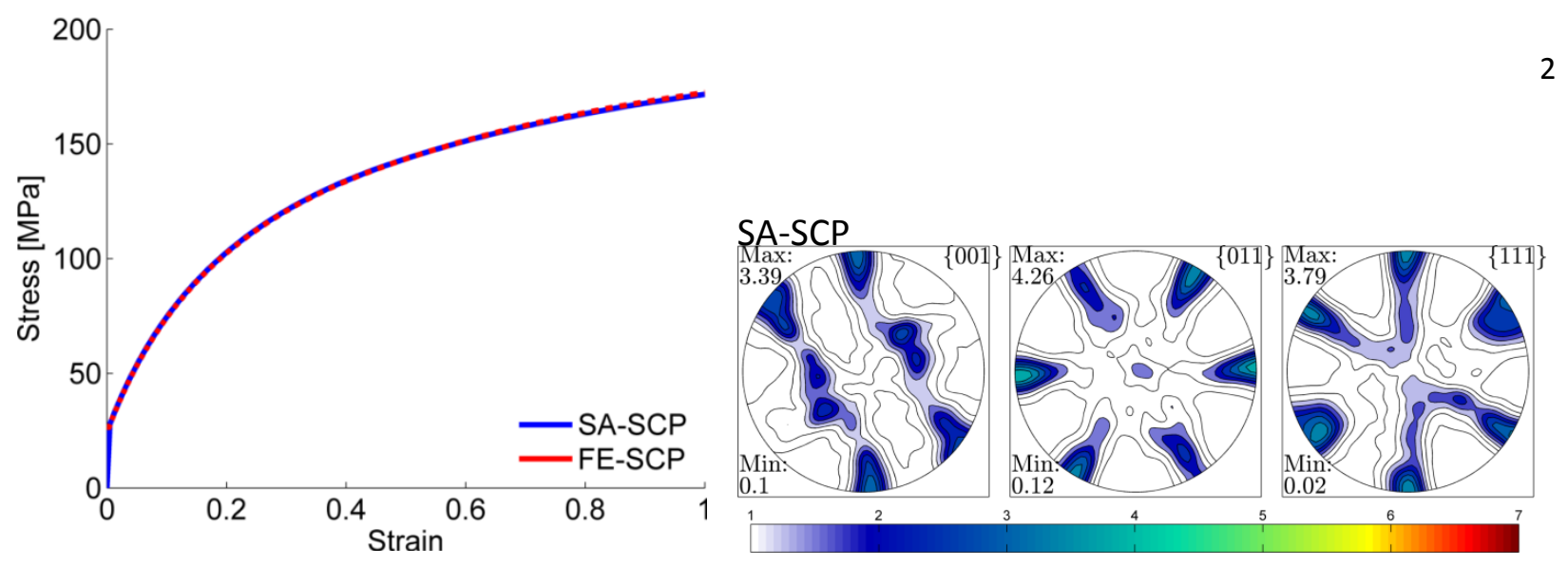

Fig. 2. Comparison of the predictions using the FE-SCP UMAT described here and the corresponding predictions using the SA-SCP model for simple shear: stress-strain curves and pole figures at a strain of 1.0.

\subsection{Simple compression of a single crystal}

In this case study, we simulate simple compression of a single crystal in $3 \mathrm{D}$ and compare the predicted geometrical changes with the published data in (Kalidindi and Anand, 1993). Figure 3a shows the orientation of the crystal relative to the reference sample frame. The compression direction, $\mathbf{e}_{3}$, is parallel with the crystallographic [110] direction. The sample $\mathbf{e}_{1}$ direction is aligned with the crystallographic [100] direction. We initialized each integration point, i.e., each material point in every element of the FE mesh with the same single crystal, as Fig. b and c show. The undeformed FE mesh is shown in Fig. 3c. The mesh consists of 2752 C3D8 elements. The geometrical changes of the samples compressed to a strain of -0.5 using the isotropic $\mathrm{J} 2$ plasticity model (Simo and Taylor, 1985) and FE-SCP are shown in Fig. 3d and e, respectively. The macroscopic shape shown in Fig. 3e shows a significant ovalization while the shape in Fig. $3 \mathrm{~d}$ is circular, which is an expected prediction by the isotropic model. The predictions shown in Fig. $3 \mathrm{e}$ are in excellent agreement with the photographed cross-section of the experimentally deformed single crystal of the same orientation presented in (Kalidindi and Anand, 1993). The dimensions of the specimen in the direction $\mathbf{e}_{2}$ remain almost unchanged meaning that the sample undergoes a plain strain compression. This is expected considering that the driving force on the slip systems in the slip direction aligned with $\mathbf{e}_{2}$ is zero (Kalidindi and Anand, 1993). 


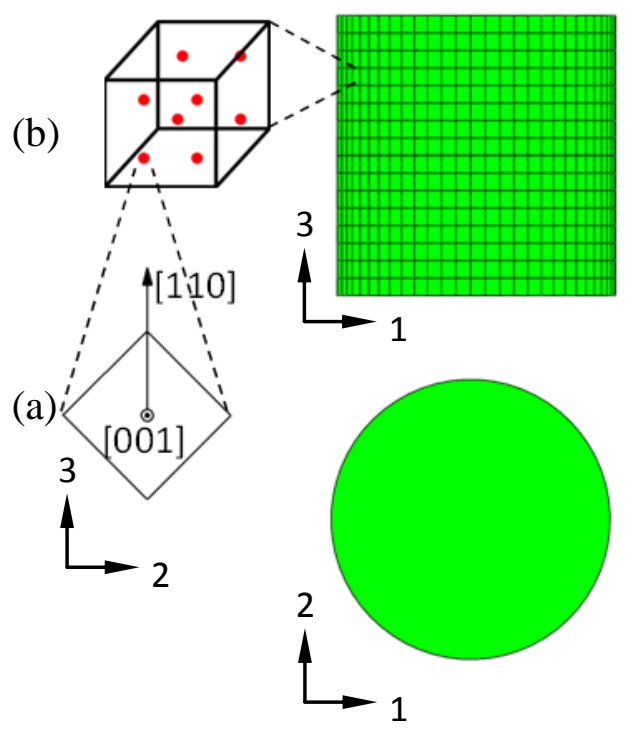

(c)

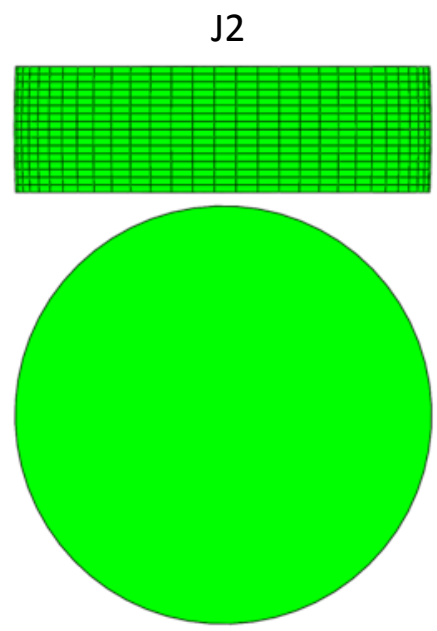

(d)

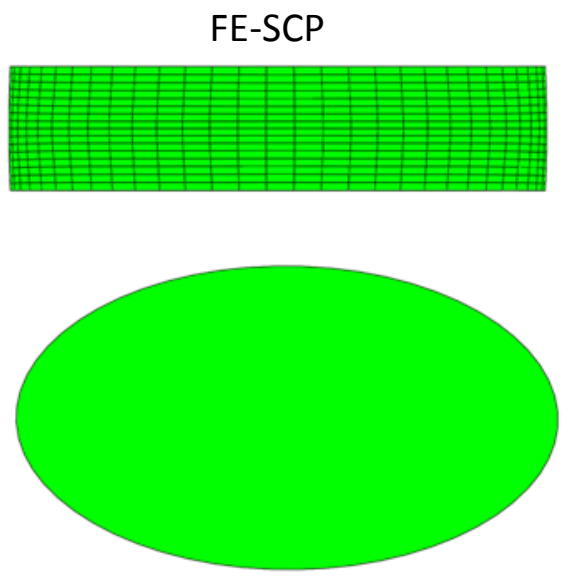

(e)

Fig. 3. Simple compression of a single crystal: (a) orientation of the crystal relative to the reference sample frame, (b) schematic showing embedding the single crystal in an integration point, (c) initial finite element mesh of 2752 C3D8 elements, (d) shape after deformation using isotropic plasticity, and (e) shape after deformation using FE-SCP UMAT.

\section{Applications of the FE-SCP UMAT}

To further illustrate the capabilities of the FE-SCP UMAT, we simulate spatial texture variations in rolling and equi-channel angular extrusion (ECAE). Both of these case studies involve large and heterogeneous deformation with ECAE involving larger strains and imposed sample rotations. Large heterogeneous strains cause texture gradients across the work piece. To capture the effect of gradients on texture evolution, we employ FE-SCP. Figure 4a and b show initial setups for the rolling and ECAE, respectively.

The rolling simulation consists of a single rolling pass resulting in a $50 \%$ reduction in thickness (from 20 to 10 in arbitrary units but we will use $\mathrm{mm}$ ) in 2D. Initial dimensions of the plate were $50 \mathrm{~mm}$ in length and $20 \mathrm{~mm}$ height. Due to the presence of orthotropic symmetry, we model half of the system and impose the symmetry boundary conditions. Deformation is applied by the rotation of the roll of a radius $R=83 \mathrm{~mm}$ with an angular velocity of $\omega=1 / \mathrm{s}$ through the contact conditions between the rolls and the plate. Initially the plate was pushed until the plate was taken by the rolls and completely rolled. The friction coefficient was assumed to be $\mu=0.4$, which was estimated based on the geometry of bite angle calculated to be $\alpha=20^{\circ}$ from the rolls 
and the height of the initial plate: $\mu>\tan (\alpha)=0.36$. We used 396 CPE4 bilinear plane strain elements to mesh the initial plate.

ECAE is a severe plastic deformation processing technique that does not change the crosssection of the sample (Beyerlein et al., 2005a). The material is extruded through a die consisting of intersecting channels that impart severe strains to the sample. We perform a $3 \mathrm{D}$, one pass ECAE simulation in a rounded die, consistent with the experimental setup and dimensions reported in (Beyerlein et al., 2005b), to demonstrate robustness of our numerical implementation and, in particular, the capability of the model to capture non-uniform texture evolution across the sample. Due to the presence of mirror symmetry, we model half of the bar and impose a symmetry boundary conditions in the $(1,2)$ plane. The billet is pushed from the top using a punch shown in red in Fig. 4. The friction coefficient between the surfaces in contact was assumed to be $\mu=0.1$ (Beyerlein et al., 2005b).

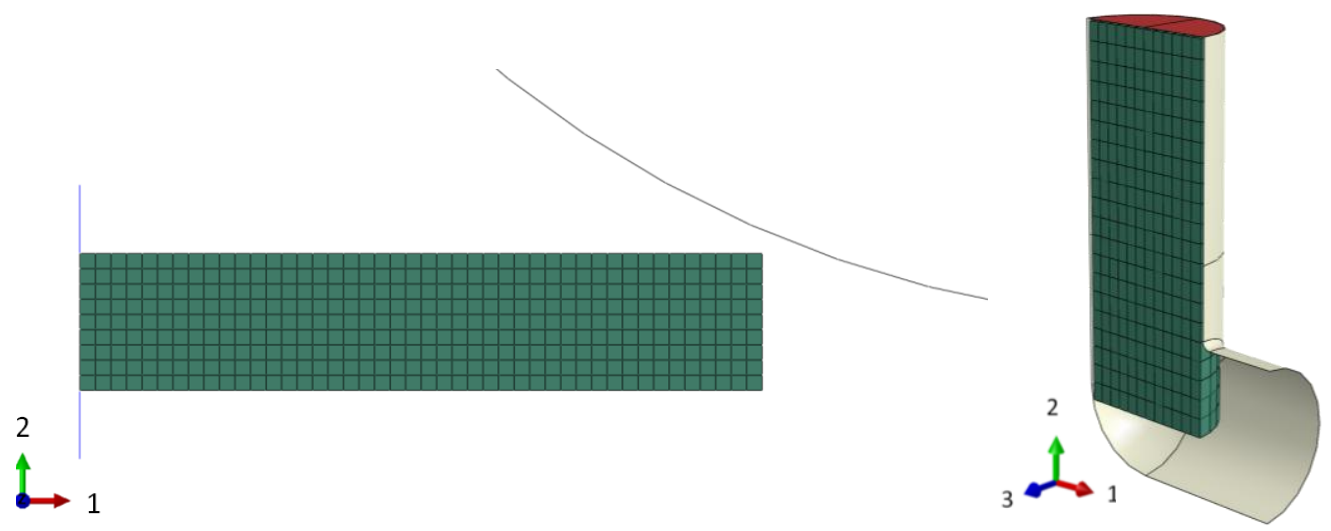

Fig. 4. Setups for the rolling simulation to a reduction of $50 \%$ and the ECAE simulation in a rounded die (Beyerlein et al., 2005b). Initial 2D mesh of the plate consists of $396 \mathrm{CPE} 4$ plane strain elements. Initial 3D mesh of the cylindrical billet consists of 1040 C3D8 elements.

Figure 5 shows the distribution of von Mises stress and equivalent plastic strain in the rolling simulation. $\mathrm{J} 2$ isotropic plasticity simulations were performed using isotropic hardening based on the macroscopic stress-strain curve. The macroscopic stress-strain curve used in the J2 simulations was the same as that used for the calibration of the hardening parameters for FESCP. The stress-strain contours predicted by J2 and FE-SCP are expected to be similar because copper has a high symmetry FCC crystal structure that does not exhibit a substantial anisotropy of material properties for textures with intensity less than 5 and can be approximated using $\mathbf{J} 2$ 
plasticity (Borja, 2013). Therefore, the stress-strain results calculated based on the J2 plasticity can be used to validate the FE-SCP model. Similar contours are indeed predicted by both the FESCP and $\mathbf{J} 2$ plasticity models. However due to texture evolution captured by the FE-SCP model, material anisotropy develops and, therefore, some discrepancy in contour plots predicted by $\mathrm{J} 2$ and FE-SCP is expected. The benefits of the FE-SCP model are in the prediction of texture evolution and associated development of anisotropy in the material response. Upon exiting the rolls, the elements near the surface have undergone significant shearing. This shearing is known to be the consequence of the friction between the contacting rolls and plate surfaces. The polycrystalline material points near the surface experience different deformation histories than the points at the center of the plate. The deformation histories determine the evolution of the texture. Texture evolution represented as $\{111\}$ pole figures near the surface and near the center are shown in the Fig. 5. The initial texture was assumed to be random and numerically approximated by a set of 512 discrete crystal orientations. The predicted texture near the center resembles a typical texture obtained under plane strain conditions (Beyerlein et al., 2005a), while the texture predicted near the surface contains a combination of the typical texture components obtained under plain strain and simple shear conditions (Kocks et al., 1998).

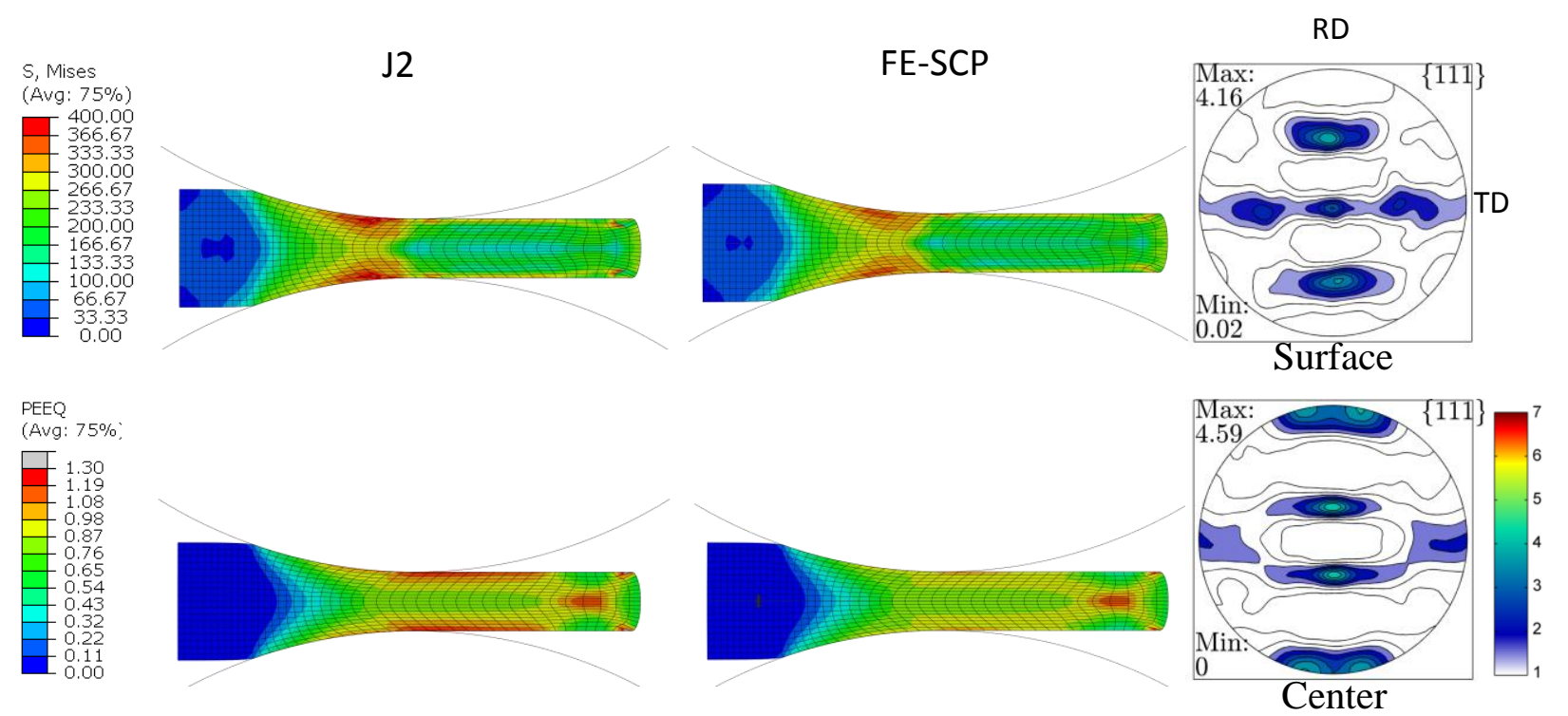

Fig. 5. Fields of von Mises stress in $[\mathrm{MPa}]$ and equivalent plastic strain as predicted by the isotropic J2 plasticity and the FE-SCP model presented here during rolling. $\{111\}$ pole figures showing predicted texture by FE-SCP after 50\% reduction at two polycrystalline material points: near the surface and near the center of the plate. 
As a final example, we present a simulation of texture evolution in a more complex nonmonotonic deformation process. We performed the simulations again using $\mathrm{J} 2$ plasticity and our new FE-SCP model. The deformed meshes showing the von Mises stress and equivalent plastic strain are presented in the Fig. 6. It is seen that the primary non-uniformity in the strain distribution is between the upper and bottom parts of the billet. In particular, the bottom experiences substantially less plastic strain. We show predicted texture corresponding to the deformation history at three different locations in the billet: one close to the top, one at the middle, and one close to the bottom of the billet. The initial texture was assumed to be random and numerically approximated by a set of 512 discrete crystal orientations. The $\{111\}$ pole figures of predicted textures using the FE-SCP model at the selected locations in the billet after one pass of ECAE are in excellent agreement with the experimentally measured textures in (Beyerlein et al., 2005a).

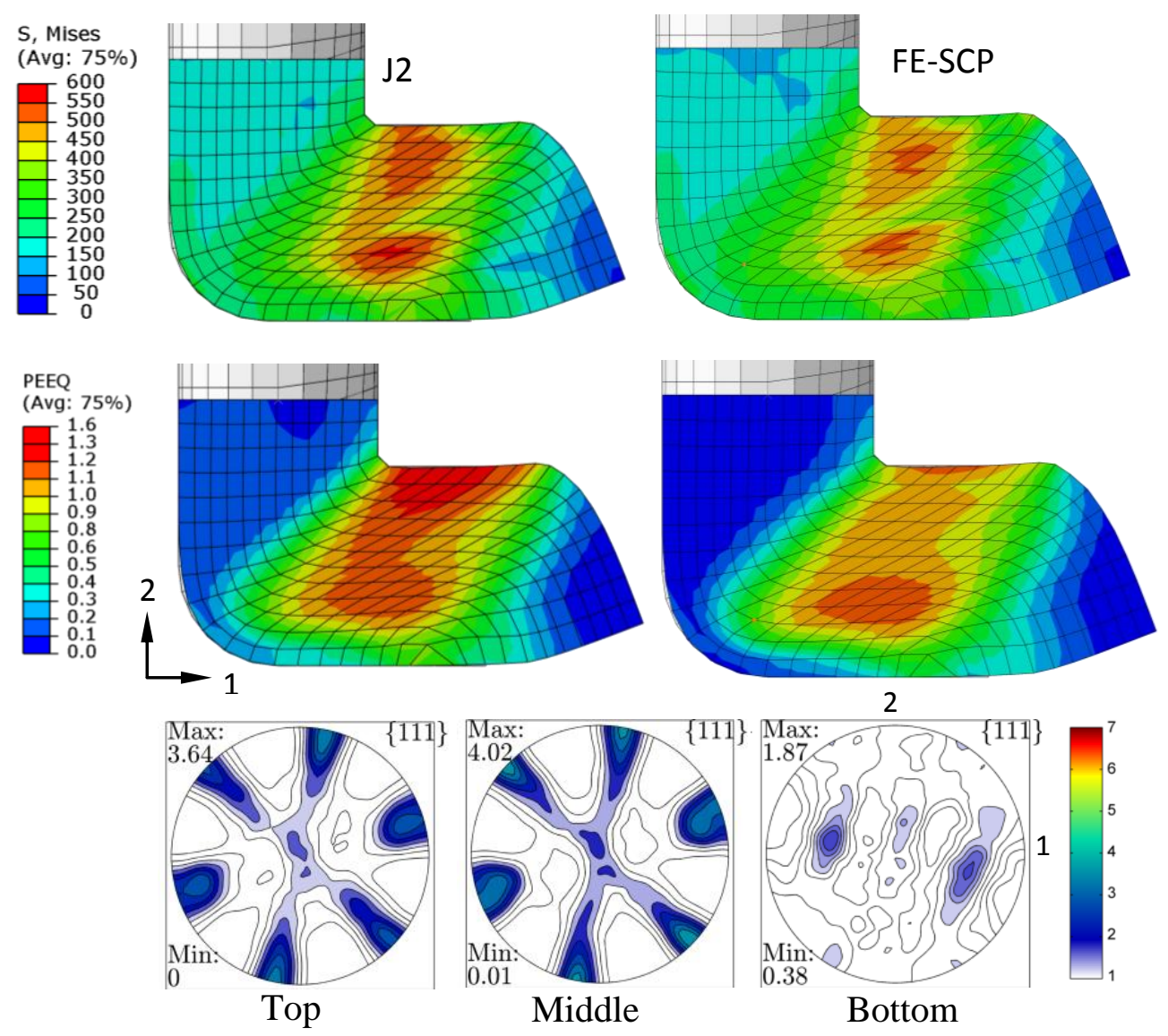


Fig. 6. Fields of von Mises stress in $[\mathrm{MPa}]$ and equivalent plastic strain as predicted by the isotropic $\mathbf{J} 2$ plasticity and the FE-SCP model presented here during ECAE. $\{111\}$ pole figures showing predicted texture by FE-SCP after the extrusion at three polycrystalline material points: near the top surface, near the center, and near the bottom of the billet.

\subsection{Conclusions}

In this paper, spectral crystal plasticity is successfully coupled with the implicit version of the FE package ABAQUS through a user material subroutine, UMAT, in ABAQUS to provide the constructive relationship between stress and strain at each FE integration point. We present the derivation of a fully analytical Jacobian matrix providing a fast convergence. An FE integration point represents either a single crystal or a polycrystal with a given initial texture that evolves with plastic deformation. We validate and apply the FE-SCP on several simulations involving FCC pure copper. Specifically, we show that the model captures the mechanical response and texture evolution under uniaxial compression and simple shear, geometrical changes under compression of a single crystal, and gradients in texture evolution under rolling and ECAE. The latter applications illustrate the potential of the FE-SCP model. Future work will combine the FE-SCP UMAT with the implementation of the SCP on hybrid CPU-GPU hardware (Knezevic and Savage, 2014).

\section{Acknowledgements}

M.Z. acknowledges support from the CEPS Graduate Fellowships program at the University of New Hampshire (UNH). M.K. acknowledges subcontract, NO. 277871, granted by Los Alamos National Laboratory to UNH. The work was supported by the US Department of Energy under Contract No. DE-AC52-06NA25396.

\section{Appendix A}

Derivative of Cauchy deviatoric stress with respect to the Cauchy stress, $\frac{\partial \boldsymbol{\sigma}^{\prime}}{\partial \boldsymbol{\sigma}}$, is:

$$
\frac{\partial \boldsymbol{\sigma}^{\prime}}{\partial \boldsymbol{\sigma}}=\left[\begin{array}{cccccc}
2 / 3 & -1 / 3 & -1 / 3 & 0 & 0 & 0 \\
-1 / 3 & 2 / 3 & -1 / 3 & 0 & 0 & 0 \\
0 & 0 & 0 & 1 & 0 & 0 \\
0 & 0 & 0 & 0 & 1 & 0 \\
0 & 0 & 0 & 0 & 0 & 1
\end{array}\right]
$$


using,

$$
\sigma_{11}^{\prime}=\frac{2}{3} \sigma_{11}-\frac{1}{3} \sigma_{22}-\frac{1}{3} \sigma_{33}, \sigma_{22}^{\prime}=\frac{2}{3} \sigma_{22}-\frac{1}{3} \sigma_{11}-\frac{1}{3} \sigma_{33}, \sigma_{12}^{\prime}=\sigma_{12}, \sigma_{13}^{\prime}=\sigma_{13}, \sigma_{23}^{\prime}=\sigma_{23} \text {. }
$$

\section{Appendix B}

Here, we present details of the analytical derivative of the deviatoric stress in the sample reference frame with respect to the isochoric strain rate. The derivative is obtained using the following chain rule:

$\frac{\partial \boldsymbol{\sigma}^{\prime}}{\partial \dot{e}_{v p}}=\frac{\partial \mathbf{Q}}{\partial \dot{e}_{v p}} \widetilde{\boldsymbol{\sigma}}^{\prime} \mathbf{Q}^{T}+\mathbf{Q} \frac{\partial \widetilde{\boldsymbol{\sigma}}^{\prime}}{\partial \dot{\mathrm{e}}_{v p}} \mathbf{Q}^{T}+\mathbf{Q} \widetilde{\boldsymbol{\sigma}}^{\prime} \frac{\partial \mathbf{Q}^{T}}{\partial \dot{e}_{v p}}$.

From Eq. 5, we can calculate $\frac{\partial \widetilde{\sigma}^{\prime}}{\partial \dot{\mathbf{e}}_{v p}}$ as follows:

$$
\frac{\partial \widetilde{\boldsymbol{\sigma}}^{\prime}}{\partial \dot{\mathbf{e}}_{v p}}=\left(\frac{\partial \widetilde{\boldsymbol{\sigma}}^{\prime}}{\partial \dot{\varepsilon}} \frac{\partial \dot{\varepsilon}}{\partial \dot{\mathbf{e}}_{v p}}\right)+\left(\frac{\partial \widetilde{\boldsymbol{\sigma}}^{\prime}}{\partial \theta} \frac{\partial \theta}{\partial \dot{\mathbf{e}}_{v p}}\right)+\left(\frac{\partial \widetilde{\boldsymbol{\sigma}}^{\prime}}{\partial \widetilde{\varphi}_{1}} \frac{\widetilde{\varphi}_{1}}{\partial \dot{\mathbf{e}}_{v p}}\right)+\left(\frac{\partial \widetilde{\boldsymbol{\sigma}}^{\prime}}{\partial \widetilde{\Phi}} \frac{\partial \widetilde{\Phi}}{\partial \dot{\mathbf{e}}_{v p}}\right)+\left(\frac{\partial \widetilde{\boldsymbol{\sigma}}^{\prime}}{\partial \widetilde{\varphi}_{2}} \frac{\partial \widetilde{\varphi}_{2}}{\partial \dot{\mathbf{e}}_{v p}}\right)+\left(\frac{\partial \widetilde{\boldsymbol{\sigma}}^{\prime}}{\partial \mathrm{s}} \frac{\partial \mathrm{s}}{\partial \dot{\mathbf{e}}_{v p}}\right)
$$

We now describe each term in Eq. B.2. The following terms, $\frac{\partial \widetilde{\boldsymbol{\sigma}}^{\prime}}{\partial \dot{\varepsilon}}$ and $\frac{\partial \widetilde{\boldsymbol{\sigma}}^{\prime}}{\partial \mathrm{s}}$, are easy to calculate and will not be discussed. The term, $\frac{\partial \dot{\varepsilon}}{\partial \dot{\mathbf{e}}_{v p}}$, represents a derivative of the norm of a matrix with respect to the same matrix (e.g. Itskov, 2007). Differentiation of the stress deviator with respect to the angles, $\frac{\partial \widetilde{\boldsymbol{\sigma}}^{\prime}}{\partial \theta}, \frac{\partial \widetilde{\boldsymbol{\sigma}}^{\prime}}{\partial \widetilde{\varphi}_{1}}, \frac{\partial \widetilde{\boldsymbol{\sigma}}^{\prime}}{\partial \widetilde{\Phi}}$, and $\frac{\partial \widetilde{\boldsymbol{\sigma}}^{\prime}}{\partial \widetilde{\varphi}_{2}}$, is performed using standard formulas available in the FFT literature (Briggs and Henson, 1995; Brigham, 1988; Cooley and Tukey, 1965; Duhamel and Vetterli, 1990).

As described earlier, a crystal orientation in the principal frame is described using a set of Bunge-Euler angles, $\tilde{g}=\left\{\tilde{\varphi}_{1}, \widetilde{\Phi}, \tilde{\varphi}_{2}\right\}$. Similarly, the same crystal orientation can be described in the sample frame using another set of Bunge-Euler angles, $g=\left\{\varphi_{1}, \Phi, \varphi_{2}\right\}$. Given BungeEuler angles, it is trivial to calculate the corresponding orientation matrices relating the respected frames (Bunge, 1993). Let us call the transformation matrix from the principal to the crystal frame $\widetilde{\mathbf{G}}$ and the transformation matrix from the sample to the crystal frame $\mathbf{G}$. We note that $\widetilde{\mathbf{G}}=\mathbf{G}^{T} \mathbf{Q}$. However, orientation of the principal frame, $\mathbf{Q}$, varies with the strain rate because columns in $\mathbf{Q}$ are principal vectors of the strain rate. Therefore, the derivatives of Bunge-Euler angles in the principal frame with respect to the strain rate, $\frac{\partial \widetilde{\varphi}_{1}}{\partial \dot{\mathbf{e}}_{v p}}, \frac{\partial \widetilde{\Phi}}{\partial \dot{\mathbf{e}}_{v p}}$, and $\frac{\partial \widetilde{\varphi}_{2}}{\partial \dot{\mathbf{e}}_{v p}}$ are calculated using the chain rules, e.g., $\frac{\partial \widetilde{\varphi}_{1}}{\partial \dot{\mathbf{e}}_{v p}}=\frac{\partial \widetilde{\varphi}_{1}}{\partial \mathbf{Q}} \frac{\partial \mathbf{Q}}{\partial \dot{\mathbf{e}}_{v p}}$. After writing explicitly the expression $\widetilde{\mathbf{G}}=\mathbf{G}^{T} \mathbf{Q}$ and 
solving for the angles (from the crystal to the principal frame), it is easy to calculate their derivatives with respect to the eigenvectors of the strain rate, i.e. $\frac{\partial \widetilde{\varphi}_{1}}{\partial \mathbf{Q}}, \frac{\partial \widetilde{\Phi}}{\partial \mathbf{Q}}$, and $\frac{\partial \widetilde{\varphi}_{2}}{\partial \mathbf{Q}}$. Analytical expressions exist for the derivatives of the eigenvectors and the eigenvalues of a given matrix with respect to the given matrix (Carlson and Hoger, 1986; Padovani, 2000). We use these analytical expressions to calculate derivatives of the eigenvectors of the strain rate with respect to the strain rate, $\frac{\partial \mathbf{Q}}{\partial \dot{\mathbf{e}}_{v p}}$.

The dependence of the angle, $\theta$, with respect to the strain rate can be accessed using the following chain rule $\frac{\partial \theta}{\partial \dot{\mathbf{e}}_{v p}}=\frac{\partial \theta}{\partial \hat{\mathbf{e}}_{v p}} \frac{\partial \hat{\mathbf{e}}_{v p}}{\partial \tilde{\mathbf{e}}_{v p}} \frac{\partial \tilde{\mathbf{e}}_{v p}}{\partial \dot{\mathbf{e}}_{v p}}$. The first term can be calculated based on Eq. 4, the second term is the derivative of the normalized eigenvalues of the strain rate with respect to the eigenvalues of the strain rate, and the third term is the derivative of the eigenvalues of the strain rate with respect to the strain rate.

Finally, the derivative of the slip resistance, defined in Eq. 3, with respect to the strain rate is: $\frac{\partial s}{\partial \dot{e}_{v p}}=h \frac{1}{s_{s}^{a}}\left(s_{s}-s\right)^{a} \frac{\partial}{\partial \dot{e}_{v p}}\left(\Sigma\left|\dot{\gamma}^{\beta}\right|\right) \Delta t$.

From Eq. 7, we can derive the expression for the derivative of the summation of the shear rates with respect to the strain rate:

$\frac{\partial}{\partial \dot{\mathbf{e}}_{v p}}\left(\sum\left|\dot{\gamma}^{\beta}\right|\right)=\frac{\partial}{\partial \dot{\varepsilon}}\left(\sum\left|\dot{\gamma}^{\beta}\right|\right) \frac{\partial \dot{\varepsilon}}{\partial \dot{\mathbf{e}}_{v p}}+\frac{\partial}{\partial \theta}\left(\sum\left|\dot{\gamma}^{\beta}\right|\right) \frac{\partial \theta}{\partial \dot{\mathbf{e}}_{v p}}+\frac{\partial}{\partial \widetilde{\varphi}_{1}}\left(\sum\left|\dot{\gamma}^{\beta}\right|\right) \frac{\partial \widetilde{\varphi}_{1}}{\partial \dot{\mathbf{e}}_{v p}}+\frac{\partial}{\partial \widetilde{\Phi}}\left(\sum\left|\dot{\gamma}^{\beta}\right|\right) \frac{\partial \widetilde{\Phi}}{\partial \dot{\mathbf{e}}_{v p}}+$

$\frac{\partial}{\partial \widetilde{\varphi}_{2}}\left(\sum\left|\dot{\gamma}^{\beta}\right|\right) \frac{\partial \widetilde{\varphi}_{2}}{\partial \dot{\mathbf{e}}_{v p}}$.

It should be recognized that all of the derivatives in Eq. B.4 have already been defined except $\frac{\partial}{\partial \theta}\left(\sum\left|\dot{\gamma}^{\beta}\right|\right), \frac{\partial}{\partial \widetilde{\varphi}_{1}}\left(\sum\left|\dot{\gamma}^{\beta}\right|\right), \frac{\partial}{\partial \widetilde{\Phi}}\left(\sum\left|\dot{\gamma}^{\beta}\right|\right)$, and $\frac{\partial}{\partial \widetilde{\varphi}_{2}}\left(\sum\left|\dot{\gamma}^{\beta}\right|\right)$, which can be readily obtained.

\section{Appendix C}

Overall algorithm of the FE-SCP UMAT implementation:

1. If time $=0$ : Initialize arrays for texture by reading a set of crystal orientations from a file (initial Bunge-Euler angles), arrays for slip resistances, and arrays of Fourier transforms by loading the spectral database from files.

Else: Initialize arrays for texture and slip resistances from the ABAQUS state variables array. 
2. Initialize stress from the previous increment and perform a rotation for $\Delta \mathbf{R}$ of stress, $\boldsymbol{\sigma}^{t, r o t}=\Delta \mathbf{R}^{*} \boldsymbol{\sigma}^{t} \Delta \mathbf{R}^{*}$, and texture.

3. Calculate elastic stiffness, $\mathbf{C}$, and elastic compliance, $\mathbf{S}$.

4. Enter Newton's method with an initial guess $\mathbf{Y}^{0}$, which is the solution of the previous increment and exit with a solution for the current increment, $\mathbf{Y}^{k}$, and updated slip resistances $s^{t+\Delta t}$.

5. If elastic behavior, calculate stress and Jacobian according to: $\boldsymbol{\sigma}^{t+\Delta t}=\boldsymbol{\sigma}^{t}+\mathbf{C} \cdot \boldsymbol{\Delta} \boldsymbol{\varepsilon}$ and $\frac{\partial \Delta \boldsymbol{\sigma}}{\partial \Delta \boldsymbol{\varepsilon}}=\mathbf{C}$, go to 7

6. Calculate the plastic spin, $\mathbf{W}^{p}\left(\dot{\boldsymbol{\varepsilon}}_{v p}^{t+\Delta t}\right)$, using Eq. 6 and reorient texture; calculate stress and Jacobian using: $\boldsymbol{\sigma}^{t+\Delta t}=\boldsymbol{\sigma}^{\prime}\left(\dot{\boldsymbol{\varepsilon}}_{v p}^{t+\Delta t}\right)+\sigma_{m}^{t+\Delta t} \mathbf{I}$ and $\frac{\partial \Delta \boldsymbol{\sigma}}{\partial \Delta \boldsymbol{\varepsilon}}=\left(\mathbf{S}+\frac{\partial \dot{\boldsymbol{\varepsilon}}_{v p}}{\partial \dot{\mathbf{e}}_{v p}}\left(\frac{\partial \boldsymbol{\sigma}^{\prime}}{\partial \dot{\mathbf{e}}_{v p}}\right)^{-1} \frac{\partial \boldsymbol{\sigma}^{\prime}}{\partial \boldsymbol{\sigma}} \Delta t\right)^{-1}$.

7. Update the ABAQUS state variables array with new crystal orientations and slip resistances.

Newton's method:

Enter the method with the initial guess, $\mathbf{Y}^{0}$, choose a step length $\lambda^{0}=1$ and calculate the first residual $\mathbf{X}\left(\mathbf{Y}^{0}\right)$.

For $\mathrm{k}=0,1, .$. do:

1. If $\mathbf{X}\left(\mathbf{Y}^{k}\right) \leq$ tolerance : exit with $\mathbf{Y}^{k}$ as solution.

Else: Calculate the Jacobian matrix $\mathbf{J}_{N R}\left(\mathbf{Y}^{\mathrm{k}}\right)=\frac{\partial \mathbf{X}}{\partial \mathbf{Y}}\left(\mathbf{Y}^{\mathrm{k}}\right)$ and obtain a correction $\Delta \mathbf{Y}^{\mathrm{k}}=-\mathbf{J}_{N R}^{-1}\left(\mathbf{Y}^{k}\right) \mathbf{X}\left(\mathbf{Y}^{k}\right)$

2. If $\lambda^{k}<\lambda_{\text {min }}=4 \cdot 10^{-7}$, calculate a new initial guess $\mathbf{Y}^{0}$, start over.

Else: Calculate new values for the solution $\mathbf{Y}^{k+1}=\mathbf{Y}^{k}+\lambda^{k} \Delta \mathbf{Y}^{k}$, the residual $\mathbf{X}\left(\mathbf{Y}^{k+1}\right)$, and a contraction factor $\theta^{k}=\frac{\left|\mathbf{X}\left(\mathbf{Y}^{k+1}\right)\right|}{\left|\mathbf{X}\left(\mathbf{Y}^{k}\right)\right|}$. 
3. If $\theta^{k} \geq 1: \lambda^{k}=\frac{1}{2} \lambda^{k}$, go to 2 .

Else: If $\left|\dot{\mathbf{e}}_{v p}^{k+1}\right| \Delta t \leq|\Delta \varepsilon| \cdot 10^{-6}$ : assume elastic behavior and exit.

Else: Accept iterate, set $k \rightarrow k+1$ and go to 1 .

\section{References}

2013. ABAQUS Version 6.11-2, Dassault Systèmes, Providence, RI, USA.

Adams, B.L., Olson, T., 1998. Mesostructure - properties linkage in polycrystals. Progress in Materials Science 43, 1-88.

Al-Harbi, H.F., Kalidindi, S.R., Crystal plasticity finite element simulations using a database of discrete Fourier transforms. International Journal of Plasticity DOI: 10.1016/j.ijplas.2014.04.006.

Al-Harbi, H.F., Knezevic, M., Kalidindi, S.R., 2010. Spectral Approaches for the Fast Computation of Yield Surfaces and First-Order Plastic Property Closures for Polycrystalline Materials with Cubic-Triclinic Textures. CMC: Computers, Materials, \& Continua 15, 153172.

Ardeljan, M., Beyerlein, I.J., Knezevic, M., 2014. A dislocation density based crystal plasticity finite element model: Application to a two-phase polycrystalline HCP/BCC composites. Journal of the Mechanics and Physics of Solids 66, 16-31.

Armijo, L., 1966. Minimization of functions having Lipschitz continuous first partial derivatives. Pacific Journal of mathematics 16, 1-3.

Asaro, R.J., Needleman, A., 1985. Texture development and strain hardening in rate dependent polycrystals. Acta Metallurgica et Materialia 33, 923-953.

Balasubramanian, 1996. Single crystal and polycrystal elasto-viscoplasticity: Application to earing in cup drawing of F.C.C. materials. Computational Mechanics 17., 209-225.

Barton, N., Bernier, J., Knap, J., Sunwoo, A., Cerreta, E., Turner, T., 2011. A call to arms for task parallelism in multi-scale materials modeling. Int. J. Numer. Meth. Engng. 86, 744-764.

Barton, N.R., Knap, J., Arsenlis, A., Becker, R., Hornung, R.D., Jefferson, D.R., 2008. Embedded polycrystal plasticity and adaptive sampling. International Journal of Plasticity 24, 242-266.

Bathe, K.-J., 1996. Finite element procedures. Englewood Cliffs, N.J.: Prentice Hall.

Beaudoin, A.J., Dawson, P.R., Mathur, K.K., Kocks, U.F., Korzekwa, D.A., 1994. Application of polycrystal plasticity to sheet forming. Computer Methods in Applied Mechanics and Engineering 117, 49-70.

Beaudoin, A.J., Mathur, K.K., Dawson, P.R., Johnson, G.C., 1993. Three-Dimensional Deformation Process Simulation With Explicit Use of Polycrystal Plasticity Models. International Journal of Plasticity 9, 833-860.

Beyerlein, I., Li, S., Necker, C., Alexander, D., Tomé, C., 2005a. Non-uniform microstructure and texture evolution during equal channel angular extrusion. Philosophical Magazine 85, 1359-1394.

Beyerlein, I.J., Li, S., Necker, C.T., Alexander, D.J., Tomé, C.N., 2005b. Non-uniform microstructure and texture evolution during equal channel angular extrusion. Philosophical Magazine 85, 1359-1394. 
Beyerlein, I.J., McCabe, R.J., Tomé, C.N., 2011. Effect of microstructure on the nucleation of deformation twins in polycrystalline high-purity magnesium: A multi-scale modeling study. Journal of the Mechanics and Physics of Solids 59, 988-1003.

Borja, R.I., 2013. Plasticity Modeling \& Computation. Springer Berlin Heidelberg.

Briggs, W.L., Henson, V.E., 1995. The DFT : an owner's manual for the discrete Fourier transform. Society for Industrial and Applied Mathematics, Philadelphia.

Brigham, E.O., 1988. The Fast Fourier Transform and Applications. Prentice Hall, Englewood Cliffs.

Bunge, H.-J., 1993. Texture analysis in materials science. Mathematical Methods. Cuvillier Verlag, Göttingen.

Carlson, D.E., Hoger, A., 1986. The derivative of a tensor-valued function of a tensor. Quart. Appl. Math. 44, 409-423.

Chockalingam, K., Tonks, M.R., Hales, J.D., Gaston, D.R., Millett, P.C., Zhang, L., 2013. Crystal plasticity with Jacobian-Free Newton-Krylov. Computational Mechanics 51, 617627.

Cooley, J.W., Tukey, J.W., 1965. Algorithm for the machine computation of complex Fourier series. Mathematics of Computation 19, 297-301.

Duhamel, P., Vetterli, M., 1990. Fast Fourier Transforms: A Tutorial Review and a State of the Art. Signal Processing 19, 259-299.

Fast, T., Knezevic, M., Kalidindi, S.R., 2008. Application of microstructure sensitive design to structural components produced from hexagonal polycrystalline metals. Computational Materials Science 43, 374-383.

Frank, F.C., 1987. Orientation Mapping. Metallurgical Transactions A 19A, 403-408.

Fuentes-Cobas, L.E., Muñoz-Romero, A., Montero-Cabrera, M.E., Fuentes-Montero, L., Fuentes-Montero, M.E., 2013. Predicting the Coupling Properties of Axially-Textured Materials. Materials 6, 4967-4984.

Hill, 1966. Generalized constitutive relations for incremental deformation of metal crystals by multislip. J. Mech. Phys.Solids 14, 95-102.

Hughes, T.J., Winget, J., 1980. Finite rotation effects in numerical integration of rate constitutive equations arising in large-deformation analysis. International Journal for Numerical Methods in Engineering 15, 1862-1867.

Hutchinson, J.W., 1976. Bounds and self-consistent estimates for creep of polycrystalline materials. Proceedings of the Royal Society of London. Series A, Mathematical and Physical Sciences, 101-126.

Itskov, M., 2007. Tensor algebra and tensor analysis for engineers. Springer, Berlin.

Kalidindi, S.R., Anand, L., 1993. Large Deformation Simple Compression of a Copper SingleCrystal. Metallurgical Transactions a-Physical Metallurgy and Materials Science 24, 989992.

Kalidindi, S.R., Bronkhorst, C.A., Anand, L., 1992. Crystallographic Texture Evolution in Bulk Deformation Processing of Fcc Metals. Journal of the Mechanics and Physics of Solids 40, 537-569.

Kalidindi, S.R., Duvvuru, H.K., Knezevic, M., 2006. Spectral calibration of crystal plasticity models. Acta Materialia 54, 1795-1804.

Kalidindi, S.R., Knezevic, M., Niezgoda, S., Shaffer, J., 2009. Representation of the orientation distribution function and computation of first-order elastic properties closures using discrete Fourier transforms. Acta Materialia 57, 3916-3923. 
Knezevic, M., Al-Harbi, H.F., Kalidindi, S.R., 2009. Crystal plasticity simulations using discrete Fourier transforms. Acta Materialia 57, 1777-1784.

Knezevic, M., Beyerlein, I.J., Brown, D.W., Sisneros, T.A., Tomé, C.N., 2013a. A polycrystal plasticity model for predicting mechanical response and texture evolution during strain-path changes: Application to beryllium. International Journal of Plasticity 49, 185-198.

Knezevic, M., Beyerlein, I.J., Lovato, M.L., Tomé, C.N., Richards, A.W., McCabe, R.J., 2014a. A strain-rate and temperature dependent constitutive model for BCC metals incorporating non-Schmid effects: Application to tantalum-tungsten alloys. International Journal of Plasticity 62, 93-104.

Knezevic, M., Beyerlein, I.J., Nizolek, T., Mara, N.A., Pollock, T.M., 2013b. Anomalous Basal Slip Activity in Zirconium under High-strain Deformation. Materials Research Letters 1, 133-140.

Knezevic, M., Capolungo, L., Tomé, C.N., Lebensohn, R.A., Alexander, D.J., Mihaila, B., McCabe, R.J., 2012a. Anisotropic stress-strain response and microstructure evolution of textured $\alpha$-uranium. Acta Materialia 60, 702-715.

Knezevic, M., Carpenter, J.S., Lovato, M.L., McCabe, R.J., 2014b. Deformation behavior of the cobalt-based superalloy Haynes 25: Experimental characterization and crystal plasticity modeling. Acta Materialia 63, 162-168.

Knezevic, M., Jahedi, M., Korkolis, Y.P., Beyerlein, I.J., 2014c. Material-based design of the extrusion of bimetallic tubes. Computational Materials Science 95, 63-73.

Knezevic, M., Kalidindi, S.R., 2007. Fast computation of first-order elastic-plastic closures for polycrystalline cubic-orthorhombic microstructures. Computational Materials Science 39, 643-648.

Knezevic, M., Kalidindi, S.R., Fullwood, D., 2008a. Computationally efficient database and spectral interpolation for fully plastic Taylor-type crystal plasticity calculations of facecentered cubic polycrystals. International Journal of Plasticity 24, 1264-1276.

Knezevic, M., Kalidindi, S.R., Mishra, R.K., 2008b. Delineation of first-order closures for plastic properties requiring explicit consideration of strain hardening and crystallographic texture evolution. International Journal of Plasticity 24, 327-342.

Knezevic, M., Lebensohn, R.A., Cazacu, O., Revil-Baudard, B., Proust, G., Vogel, S.C., Nixon, M.E., 2013c. Modeling bending of $\alpha$-titanium with embedded polycrystal plasticity in implicit finite elements. Materials Science and Engineering: A 564, 116-126.

Knezevic, M., Levinson, A., Harris, R., Mishra, R.K., Doherty, R.D., Kalidindi, S.R., 2010. Deformation twinning in AZ31: Influence on strain hardening and texture evolution. Acta Materialia 58, 6230-6242.

Knezevic, M., McCabe, R.J., Lebensohn, R.A., Tomé, C.N., Liu, C., Lovato, M.L., Mihaila, B., 2013d. Integration of self-consistent polycrystal plasticity with dislocation density based hardening laws within an implicit finite element framework: Application to low-symmetry metals. Journal of the Mechanics and Physics of Solids 61, 2034-2046.

Knezevic, M., McCabe, R.J., Lebensohn, R.A., Tomé, C.N., Mihaila, B., 2012b. Finite element implementation of a self-consistent polycrystal plasticity model: Application to $\alpha$-uranium, Proceedings: Volume 2: Materials Properties, Characterization, and Modeling TMS (The Minerals, Metals \& Materials Society), pp. 789-796.

Knezevic, M., McCabe, R.J., Tomé, C.N., Lebensohn, R.A., Chen, S.R., Cady, C.M., Gray Iii, G.T., Mihaila, B., 2013e. Modeling mechanical response and texture evolution of $\alpha$-uranium 
as a function of strain rate and temperature using polycrystal plasticity. International Journal of Plasticity 43, 70-84.

Knezevic, M., Nizolek, T., Ardeljan, M., Beyerlein, I.J., Mara, N.A., Pollock, T.M., 2014d. Texture evolution in two-phase $\mathrm{Zr} / \mathrm{Nb}$ lamellar composites during accumulative roll bonding. International Journal of Plasticity 57, 16-28.

Knezevic, M., Savage, D.J., 2014. A high-performance computational framework for fast crystal plasticity simulations. Computational Materials Science 83, 101-106.

Kocks, U.F., Tome', C.N., Wenk, H.-R., 1998. Texture and Anisotropy. Cambridge University Press, Cambridge.

Kumar, A., Dawson, P., 1995. Polycrystal plasticity modeling of bulk forming with finite elements over orientation space. Computational mechanics 17, 10-25.

Lebensohn, R.A., Kanjarla, A.K., Eisenlohr, P., 2012. An elasto-viscoplastic formulation based on fast Fourier transforms for the prediction of micromechanical fields in polycrystalline materials. International Journal of Plasticity 32-33, 59-69.

Lebensohn, R.A., Tomé, C.N., 1993. A self-consistent anisotropic approach for the simulation of plastic deformation and texture development of polycrystals: Application to zirconium alloys. Acta Metallurgica et Materialia 41, 2611-2624.

Lebensohn, R.A., Tomé, C.N., Castaneda, P.P., 2007. Self-consistent modelling of the mechanical behaviour of viscoplastic polycrystals incorporating intragranular field fluctuations. Philosophical Magazine 87, 4287-4322.

Mathur, K., K., Dawson, P.R., 1989. On modelling the development of crystallographic texture in bulk forming processes. International Journal of Plasticity 5, 67-94.

Meyers, M.A., Chawla, K.K., 1998. Mechanical Behavior of Materials. Prentice Hall, Upper Saddle River, New Jersey.

Mihaila, B., Knezevic, M., Cardenas, A., 2014. Three orders of magnitude improved efficiency with high-performance spectral crystal plasticity on GPU platforms. International Journal for Numerical Methods in Engineering 97, 785-798.

Neumann, P., 1991. Representation of orientations of symmetrical objects by Rodrigues vectors. Textures and Microstructures 14-18, 53-58.

Padovani, C., 2000. On the derivative of some tensor-valued functions. Journal of elasticity and the physical science of solids 58, 257-268.

Peirce, D., Asaro, R., Needleman, A., 1982. An analysis of nonuniform and localized deformation in ductile single crystals. Acta metallurgica 30, 1087-1119.

Press, W.H., Teukolsky, S. A., Vetterling, W. T., and Flannery. B. P, 2002. Numerical Recipes in $\mathrm{C}++$.

Raabe, D., Roters, F., 2004. Using texture components in crystal plasticity finite element simulations. International Journal of Plasticity 20, 339-361.

Roters, F., Eisenlohr, P., Hantcherli, L., Tjahjanto, D.D., Bieler, T.R., Raabe, D., 2010. Overview of constitutive laws, kinematics, homogenization and multiscale methods in crystal plasticity finite-element modeling: Theory, experiments, applications. Acta Materialia 58, 1152-1211.

Sarma, G.B., Dawson, P.R., 1996. Texture predictions using a polycrystal plasticity model incorporating neighbor interactions. International Journal of Plasticity 12, 1023-1054.

Segurado, J., Lebensohn, R.A., Llorca, J., Tomé, C.N., 2012. Multiscale modeling of plasticity based on embedding the viscoplastic self-consistent formulation in implicit finite elements. International Journal of Plasticity 28, 124-140. 
Shaffer, J.B., Knezevic, M., Kalidindi, S.R., 2010. Building texture evolution networks for deformation processing of polycrystalline fcc metals using spectral approaches: Applications to process design for targeted performance. International Journal of Plasticity 26, 1183-1194.

Simo, J.C., Taylor, R.L., 1985. Consistent tangent operators for rate-independent elastoplasticity. Computer Methods in Applied Mechanics and Engineering 48, 101-118.

Sundararaghavan, V., Zabaras, N., 2007. Linear analysis of texture-property relationships using process-based representations of Rodrigues space. Acta Materialia 55, 1573-1587.

Takahashi, Y., Miyazawa, K.i., Mori, M., Ishida, Y., 1985. Quaternion representation of the orientation relationship and its application to grain boundary problems. Trans. Jpn. Inst. Met. $27,345-352$.

Taylor, G.I., 1938. Plastic strain in metals. Journal of the Institute of Metals 62, 307-324.

Tomé, C., Canova, G.R., Kocks, U.F., Christodoulou, N., Jonas, J.J., 1984. The relation between macroscopic and microscopic strain hardening in F.C.C. polycrystals. Acta Metallurgica 32, 1637-1653.

Van Houtte, P., 1994. Application of plastic potentials to strain rate sensitive and insensitive anisotropic materials. International Journal of Plasticity 10, 719-748.

Van Houtte, P., Li, S., Engler, O., 2004. Taylor-Type Homogenization Methods for Texture and Anisotropy. Continuum Scale Simulation of Engineering Materials: FundamentalsMicrostructures-Process Applications, 459-472.

Voce, E., 1948. The Relationship between Stress and Strain for homogeneous deformation. Journal of the Institute of Metals 74, 537-562. 